\title{
Stability Analysis of Bilateral Teleoperation with Bounded and Monotone Environments via Zames-Falb Multipliers
}

DOI:

10.1109/TCST.2016.2601289

\section{Document Version}

Accepted author manuscript

Link to publication record in Manchester Research Explorer

\section{Citation for published version (APA):}

Tugal, H., Carrasco, J., Falcon, P., \& Barreiro, A. (2017). Stability Analysis of Bilateral Teleoperation with Bounded and Monotone Environments via Zames-Falb Multipliers. IEEE Transactions on Control Systems Technology, 25(4), 1331. https://doi.org/10.1109/TCST.2016.2601289

\section{Published in:}

IEEE Transactions on Control Systems Technology

\section{Citing this paper}

Please note that where the full-text provided on Manchester Research Explorer is the Author Accepted Manuscript or Proof version this may differ from the final Published version. If citing, it is advised that you check and use the publisher's definitive version.

\section{General rights}

Copyright and moral rights for the publications made accessible in the Research Explorer are retained by the authors and/or other copyright owners and it is a condition of accessing publications that users recognise and abide by the legal requirements associated with these rights.

\section{Takedown policy}

If you believe that this document breaches copyright please refer to the University of Manchester's Takedown Procedures [http://man.ac.uk/04Y6Bo] or contact uml.scholarlycommunications@manchester.ac.uk providing relevant details, so we can investigate your claim.

\section{OPEN ACCESS}




\title{
Stability Analysis of Bilateral Teleoperation with Bounded and Monotone Environments via Zames-Falb Multipliers
}

\author{
Harun Tugal $^{1}$, Joaquin Carrasco ${ }^{1}$, Pablo Falcon ${ }^{2}$, Antonio Barreiro ${ }^{2}$
}

\begin{abstract}
This paper provides less conservative stability conditions for bilateral teleoperation by exploiting the advantages of the integral quadratic constraint (IQC) framework, where the environment can be defined as a memoryless, bounded, and monotonic nonlinear operator. Recent advances in multiplier theory for appropriate classes of uncertainties/nonlinearities are applied. Since the classes of multipliers have infinite dimension, parametrization of these multipliers is used to obtain convex searches over a finite number of parameters. The stability of the system is analysed as a Lurye system containing timedelay and monotone nonlinearity. As a result, less conservative delay-dependent conditions can be developed. These results are then applied to bilateral teleoperation. Finally, stability results are tested with different experiments; in particular bilateral teleoperation experiments over the internet between Manchester, UK, and Vigo, Spain, have been carried out. The advantage of the proposed approach is demonstrated by reaching higher transparency index for 2-channel position-force teleoperation while ensuring absolute stability.
\end{abstract}

\section{INTRODUCTION}

Teleoperation is the remote control of a robot through a communication channel. Usually the operator relies solely on visual information delivered by a camera. By contrast, in bilateral teleoperation, both haptic and visual information is transmitted so that the operator feels the interaction force between the remote manipulator and the environment while viewing the scene. This additional feedback is crucial in tasks requiring delicate action such as telesurgery [1], which need more steps than in, say, picking and placing, and in tasks where the environment is hazardous such as space missions, undersea exploration, and nuclear decommissioning [2], [3].

A bilateral teleoperation needs to fulfil two key criteria: absolute stability which needs to hold for any possible human and environment pairs, and transparency defining how well the environmental interaction forces or its impedances are being transmitted to the operator side. Unfortunately, there exists a trade off between these two measures and with highly variable uncertainties the overall control design process transforms into a challenging task [4]. Over the years there have been many efforts to overcome these obstacles that

\footnotetext{
${ }^{1}$ Control Systems Centre,School of Electrical and Electronic Engineering, The University of Manchester, M13 9PL, UK.

harun.tugal@manchester.ac.uk

joaquin.carrascogomez@manchester.ac.uk

${ }^{2}$ School of Industrial Engineering, University of Vigo, 36310, Vigo, Spain.

pfalcon@uvigo.es, abarreiroduvigo.es
}

prevent physically realisable high-performance teleoperation system, and passivity theory became the main tool that has been used by many researchers [5]-[8].

Passivity is an energy related phenomenon; loosely speaking a system is said to be passive if the energy being extracted from its output ports is not higher than the energy being injected into its input ports [9]. Passivity is independent of the model parameters which makes it appropriate for the teleoperation as design contains highly variable uncertainties. Therefore, the stability analyses in bilateral teleoperation are performed by transforming overall system in a network consisting one port passive human-environment pair connected to two ports network containing manipulators and controllers dynamics, etc. Under the passivity assumption of the operators, if we can somehow guarantee that two port network is also passive, then stability of the interconnection is ensured. Under the assumption that human and environment behave as Linear Time Invariant (LTI) passive operators, Llewellyn's conditions [10] become necessary and sufficient for stability of the interconnection. The price to pay for these convenient assumptions is conservatism of the design as it needs to be robust against a wide range of uncertainty classes. As a consequence, absolute stability of a 2-channel positionforce architecture with simple Proportional-Derivative (PD) controller at the slave side cannot be guaranteed [11], [12]. To reduce conservatism, the definition of the class of environments can be restricted to bounded stiffness instead of the full class of passive systems, thus reasonable performance specifications were obtained [13], [14].

In addition, master and slave manipulators are generally located at different places in bilateral teleoperation, so that latency occurs throughout communication medium. There have been a number of efforts to improve performance and recover stability of the teleoperation while communication channel accommodates a constant or time varying delay: wave variables and scattering transformation paved the way for stable time delayed teleoperation by passivation of the communication channel [15]-[20]. Less conservative results are also obtained with controller's passivity property [21], [22]. Techniques that analyse stability against an existence delay are divided in two categories; delay-independent, where stability margin is independent from the delay itself, and delay-dependent, where the system is stable against a pre-determined maximum time delay. By taking into account the possible allowable time delay less conservative results are obtained for the practical applications. 
Polat and Scherer [23] showed that stability analyses of bilateral teleoperation can be carried out by using IQC framework. Stability is transformed to convex search for suitable passive multipliers that ensure Frequency Dependent Inequalities (FDI) by using equivalent Linear Matrix Inequalities (LMI) [24]. The use of IQC analysis allows us to use different models for human and environment.

In this paper, whose initial findings were presented in [25], absolute stability of the bilateral teleoperation is analysed with IQC methodology, assuming that the environmental interaction force is a monotonic bounded nonlinearity with respect to slave's speed. The controller is designed to maximise the transparency of the system. In order to obtain an analytical advantage from this assumption, we need to search over the class of Zames-Falb multipliers [26], [27] which are the widest available class of multipliers [28] for this class of nonlinearity. Recent interest in this sort of nonlinear force feedback has been also proposed in [29]. We have extended the results in [25] by including time-delay operators and real experiments have been carried out between two remote labs. Moreover, a more convenient parametrization for the ZamesFalb multipliers is used. As intermediate result, we show that the use of IQC analysis for time-delay systems with saturated actuation provide competitive results in comparison with other techniques in the literature. The proposed methodology has wide range of application area, see [30], yet we motivate our attention in to the bilateral teleoperation.

\section{Problem Statement And Contribution}

The main concern in bilateral teleoperation is how to ensure that absolute stability is assured while obtaining sufficient performance specifications which are mainly transparency and position tracking of the slave manipulator. The methodological approach to define uncertain human and environment pair, and delay in communication channel plays crucial role. In order to use robust control techniques for stability analysis, the bilateral teleoperation architecture proposed in Fig. 1 will be transformed into classical nominal plant-uncertainty interconnection without loss of generality.

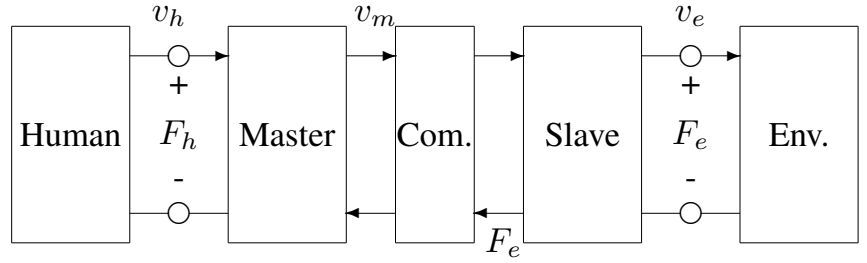

Fig. 1. Network representation of the bilateral teleoperation, where human and environment are defined as a one port and the rest is depicted as two port network.

Network representation of the system can be any of the immitance matrices; impedance, admittance or hybrid [31], in our analyses admittance matrix $\mathbf{Y}$ is used:

$$
\left[\begin{array}{c}
v_{h} \\
-v_{e}
\end{array}\right]=\left[\begin{array}{ll}
y_{11} & y_{12} \\
y_{21} & y_{22}
\end{array}\right]\left[\begin{array}{c}
F_{h} \\
F_{e}
\end{array}\right]
$$

where $F_{h}, F_{e}, v_{h}$, and $v_{e}$ are the forces and velocities of human and environment at interaction interfaces. The admittance matrix $Y(j \omega)$ substitutes nominal system $G$ in the Lurye ${ }^{1}$ structure.

Strictly speaking, the stability analysis of the position-force bilateral teleoperation, illustrated in Fig. 2a, will be analysed with two different slave controllers which are frequently used in the literature; proportional $(\mathrm{P})$ or proportional-derivative (PD) controller while trying to maximize performance criteria, transparency and position tracking.

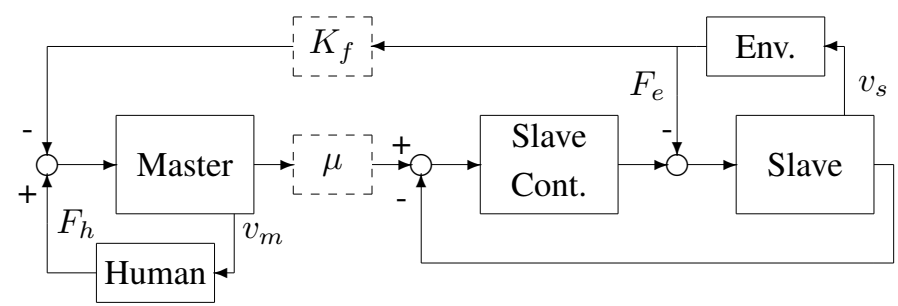

(a) Generic interconnection

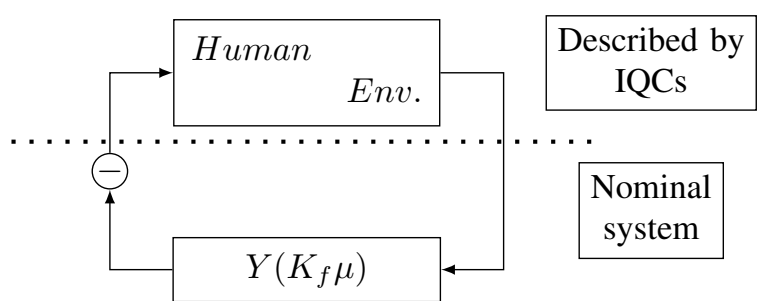

(b) Equivalent Lurye structure

Fig. 2. Delay free 2 channel Position-Force bilateral teleoperation system, where $K_{f}$ and $\mu$ are force and position scaling factors, respectively.

A physical object is said to be transparent if the light passing through it is not being scattered. In bilateral teleoperation, generally speaking, instead of light the environment's impedance or force is transmitted. And transparency is measured by defining how well the impedance or force is being transmitted. For example in the position-force bilateral teleoperation architecture level of the transparency is measured with maximum achievable force and position scaling factors, $K_{f}$ and $\mu$ respectively. The product of these two constants $\left(K_{f} \mu\right)$ will be referred to as transparency index, and it is equal to 1 in ideal conditions ${ }^{2}$. But due to the previously mentioned trade off, it is not possible to obtain an absolutely stable and also ideally transparent teleoperator [4]. Our design specification is to obtain maximum achievable transparency indexes with stable position-force architectures controlled by $\mathrm{P}$ and PD controllers. In the context of teleoperation, to the best of authors' knowledge there is no research that uses such an assumption about environmental uncertainty with Zames-Falb multiplier search as a stability criteria for bilateral teleoperation which is re-characterized as Fig. 2 b.

Time delay is inevitable phenomenon in teleoperation if two manipulators are located in different places and depending on the communication medium, delay in Fig. 3a can be constant or time-variable. To analyse that circumstance, besides human and environment latency in the communication medium is treated as an uncertainty by pulling out delays from the admittance matrix $Y\left(K_{f} \mu\right)$ and redefining nominal system

\footnotetext{
${ }^{1}$ Also written as Lurie or Lur'e.

${ }^{2}$ When master and slave manipulators are in similar dimension
} 
as $\bar{Y}\left(K_{f} \mu\right)$, see Fig. 3b. Further descriptions about these system's representations will be depicted in section VI. For comparison analyses are based on both retarded and delay free scenarios.

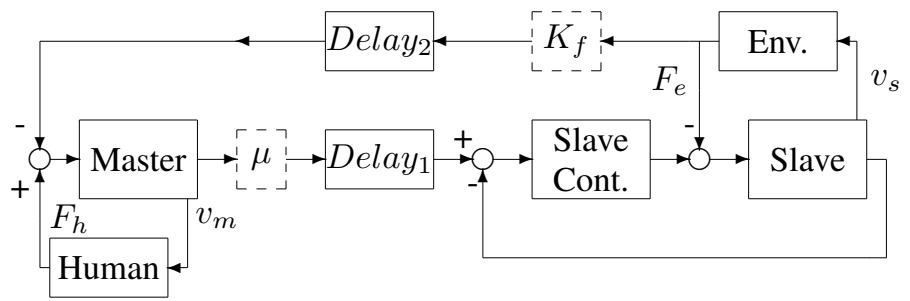

(a) Generic interconnection with time delay

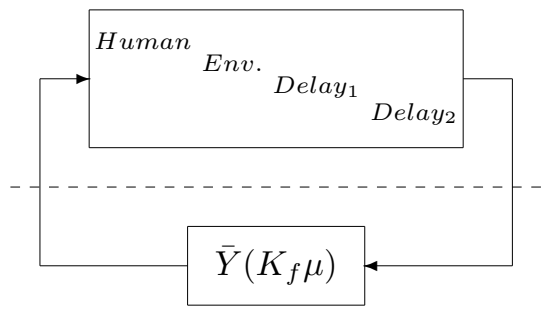

(b) Lurye structure with reconstructed nominal system $\bar{Y}$ and uncertainty

Fig. 3. Time delayed 2 channel Position-Force bilateral teleoperation.

The contribution of this paper is to show that an alternative description of the environment can be usefully exploited to obtain less conservative stability conditions, increasing the transparency index of the system; we use the class of monotone and bounded nonlinearity to characterise the environment. This new description is valid for viscosity-like forces, which have been widely used in teleoperation [4]. Moreover, this analysis can be used to design feedback forces as in [29]. By restricting the class of environments, we can reduce the conservatism of the analysis. IQC framework becomes the suitable tool to analyse several nonlinearities/uncertainties, in particular, it has been proposed for teleoperation in [23]. The advantages of the new description are demonstrated in the examples where the transparency index is clearly improved, and PD controllers, which cannot be guaranteed to be stable for passive environments, are now guaranteed to be stable for the new description.

The IQC notation and parametrization are given in Sections III and IV. Although initial results can be obtained by using the IQC- $\beta$ toolbox [32], new descriptions of delay multipliers have been given in the literature since the development of this toolbox. Here, development of the computational tools is shown to take advantage of the latest contributions. Moreover, we show that the IQC framework can be used to improve other stability results such as stability analysis of systems with delays and slope-restricted nonlinearity, which are used in delayed neural network literature [30], [33].

\section{NOTATION AND PRELIMINARIES}

Let $\mathcal{L}_{2}^{m}[0, \infty)$ be the Hilbert space of all square integrable functions $f:[0, \infty) \rightarrow \mathbb{R}^{m}$. Given $T \in \mathbb{R}$, a truncation of the function $f$ at $T$ is given by $f_{T}(t)=f(t), \quad \forall t \leq T$ and $f_{T}(t)=0, \quad \forall t>T$. The function $f$ belongs to the extended space $\mathcal{L}_{2 e}^{m}[0, \infty)$ if $f_{T} \in \mathcal{L}_{2}^{m}[0, \infty)$ for all $T>0$. The Fourier transform of $f$, henceforth $\hat{f}$, is given by $\hat{f}(j \omega)=$ $\int_{0}^{\infty} e^{-j \omega t} f(t) d t$. Let $C(c, r)$ be a circle in the complex plain with centre $c$ and radius $r$.

Let $(\star)$ be a space holder for the right outer factor of a quadratic form such that $(\star)^{*} M \Phi(j \omega) G(j \omega)=$ $G^{*}(j \omega) \Phi^{*}(j \omega) M \Phi(j \omega) G(j \omega)$. For LTI systems, $G(j \omega)^{*}=$ $G(-j \omega)^{\top}$, where ${ }^{\top}$ means transpose. The standard notation $\mathbf{R H}_{\infty}$ for stable real rational transfer function is used. A minimal state space realization of the transfer function, $G(j \omega)=C(j \omega I-A)^{-1} B+D$, is given with the shorthand $G \sim\left[\begin{array}{ll}A & B \\ C & D\end{array}\right]$. Given $n \geq 2$ and $\alpha>0$, let define a transfer function vector as

$$
{ }^{n} \Lambda_{\alpha}(j \omega)=\left[\begin{array}{llll}
1 & \frac{1}{j \omega+\alpha} & \cdots & \frac{1}{(j \omega+\alpha)^{n-1}}
\end{array}\right]^{\top}, \alpha>0,
$$

which will be used to parametrize the multipliers.

A nonlinearity $\phi: \mathcal{L}_{2 e}[0, \infty) \rightarrow \mathcal{L}_{2 e}[0, \infty)$ is said to be memoryless if there exists $N: \mathbb{R} \rightarrow \mathbb{R}$ such $(\phi v)(t)=$ $N(v(t))$ for all $t \in \mathbb{R}$. Henceforth we assume that $N(0)=0$. A memoryless nonlinearity $\phi$ is said to be bounded if there exists a positive constant $C$ such that $|N(x)|<C|x|$ for all $x \in \mathbb{R}$. The nonlinearity $\phi$ is said to be monotone if for any two real numbers $x_{1}$ and $x_{2}$ we have

$$
0 \leq \frac{N\left(x_{1}\right)-N\left(x_{2}\right)}{x_{1}-x_{2}} \text {. }
$$

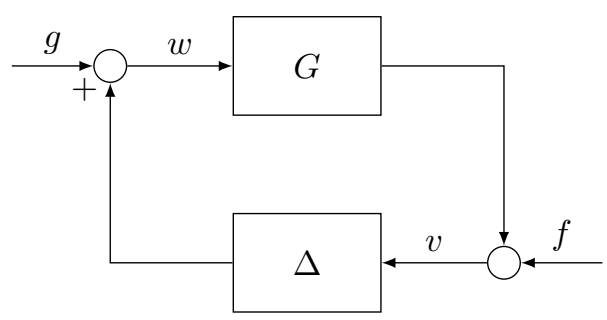

Fig. 4. Classical feedback interconnection of nominal plant and perturbation.

\section{A. IQC Framework}

Absolute stability of a teleoperation system will be transformed into the Lurye problem; positive interconnection of $G$, a linear system, and $\Delta$ which is any uncertain operator within a class of nonlinear systems. The feedback interconnection in Fig. 4 is defined by

$$
\left\{\begin{array}{l}
v=f+G w \\
w=g+\Delta v
\end{array}\right.
$$

where $g, w, \Delta v \in \mathcal{L}_{2 e}^{m}$ and $G w, v, f \in \mathcal{L}_{2 e}^{l}$. This interconnection is said to be well-posed if the map $(v, w) \mapsto(g, f)$ has a causal inverse on $\mathcal{L}_{2 e}^{m+l}[0, \infty)$. Moreover it is said to be stable if for any $(g, f) \in \mathcal{L}_{2}^{m+l}$, then $(w, v) \in \mathcal{L}_{2}^{m+l}$. The stability of the system will be analysed using the IQC framework:

Definition 1. Let $\Pi: j \mathbb{R} \rightarrow \mathbb{C}^{(l+m) \times(l+m)}$ be a Hermitian bounded measurable function. Two signals $u \in \mathcal{L}_{2}^{m}[0, \infty)$ and $y \in \mathcal{L}_{2}^{l}[0, \infty)$ are said to satisfy the IQC defined by $\Pi$, if

$$
\int_{-\infty}^{\infty}\left[\begin{array}{l}
\widehat{u}(j \omega) \\
\widehat{y}(j \omega)
\end{array}\right]^{*} \Pi(j \omega)\left[\begin{array}{l}
\widehat{u}(j \omega) \\
\widehat{y}(j \omega)
\end{array}\right] d \omega \geq 0 .
$$


Moreover, a bounded system $\Delta: \mathcal{L}_{2 e}^{m}[0, \infty) \rightarrow \mathcal{L}_{2}^{l}[0, \infty)$ is said to satisfy the IQC defined by $\Pi$ if $u$ and $\Delta u$ satisfy the IQC defined by $\Pi$ for all $u \in \mathcal{L}_{2}^{m}$.

Theorem 1 ( [34]). Let $G \in \mathbf{R H}_{\infty}^{l \times m}$, and $\Delta$ be a bounded causal operator. If the following statements hold:

1) for every $\tau \in[0,1]$, the interconnection of $G$ and $\tau \Delta$ is well-posed,

2) for every $\tau \in[0,1], \tau \Delta$ satisfies the IQC defined by $\Pi$,

3) there exist $\epsilon>0$ such that

$$
\left[\begin{array}{c}
G(j \omega) \\
I
\end{array}\right]^{*} \Pi(j \omega)\left[\begin{array}{c}
G(j \omega) \\
I
\end{array}\right] \leq-\epsilon I, \quad \forall \omega \in \mathbb{R} .
$$

Then, the positive feedback interconnection of $G$ and $\Delta$ is stable.

Remark 1. Generally, $\Pi: j \mathbb{R} \rightarrow \mathbb{C}^{(l+m) \times(l+m)}$ can be any Hermitian bounded measurable function in the form as $\left[\begin{array}{ll}\Pi_{11} & \Pi_{12} \\ \Pi_{12}^{*} \Pi_{22}\end{array}\right]$. In this paper we will restrict our attention to multipliers with $\Pi_{11} \geq 0$ and $\Pi_{22} \leq 0$ so that second condition in Theorem 1 will be satisfied if and only if $\Delta$ satisfies the IQC defined by $\Pi$.

Assume that $\Delta$ is a structured uncertainty; diagonal combination of different perturbations such that $\Delta=$ $\operatorname{diag}\left(\Delta_{1}, \ldots, \Delta_{n}\right)$ and each subsystem satisfies IQC defined by

$$
\Pi_{i}(j \omega)=\left[\begin{array}{ll}
\Pi_{i(11)} & \Pi_{i(12)}^{*} \\
\Pi_{i(12)} & \Pi_{i(22)}
\end{array}\right]
$$

where $i=1, \ldots, n$, then overall system satisfies IQC defined by [35]

$$
\Pi=\left[\begin{array}{cccc|cccc}
\Pi_{1(11)} & 0 & \cdots & 0 & \Pi_{1(12)}^{*} & 0 & \cdots & 0 \\
0 & \ddots & \ddots & \vdots & 0 & \ddots & \ddots & \vdots \\
\vdots & \ddots & \ddots & 0 & \vdots & \ddots & \ddots & 0 \\
0 & \cdots & 0 & \Pi_{n(11)} & 0 & \cdots & 0 & \Pi_{n(12)}^{*} \\
\hline \Pi_{1(12)} & 0 & \cdots & 0 & \Pi_{1(22)} & 0 & \cdots & 0 \\
0 & \ddots & \ddots & \vdots & 0 & \ddots & \ddots & \vdots \\
\vdots & \ddots & \ddots & 0 & \vdots & \ddots & \ddots & 0 \\
0 & \cdots & 0 & \Pi_{n(12)} & 0 & \cdots & 0 & \Pi_{n(22)}
\end{array}\right] .
$$

The last condition in Theorem 1 is frequency dependent infinite dimensional inequality and it will be transformed into a frequency independent finite dimensional LMI by using the Kalman-Yakubovich-Popov (KYP) Lemma:

Lemma 2 (KYP Lemma [36]). Given $A \in \mathbb{R}^{n \times n}, B \in$ $\mathbb{R}^{n \times m}, M=M^{\top} \in \mathbb{R}^{(n+m) \times(n+m)}$, with $\operatorname{det}(j \omega I-A) \neq 0$ for all $\omega$, where $[A, B]$ are controllable. The following two statements are equivalent:

1)

$$
\left[\begin{array}{c}
(j \omega I-A)^{-1} B \\
I
\end{array}\right]^{*} M\left[\begin{array}{c}
(j \omega I-A)^{-1} B \\
I
\end{array}\right] \leq 0,
$$

2) There is a matrix $P \in \mathbb{R}$ such that $P=P^{\top}$ and

$$
\left[\begin{array}{cc}
A^{\top} P+P A & P B \\
B^{\top} P & 0
\end{array}\right]+M \leq 0 .
$$

\section{B. List of IQCs}

In this section, definitions of the different classes of multipliers that we shall use in the paper are given.

An LTI system $\Delta \in \mathbf{R} \mathbf{H}_{\infty}$ is said to be passive if $\Delta(j \omega)+$ $\Delta(j \omega)^{*} \geq 0$ for all $\omega \in \mathbb{R}$. The class of multipliers preserving the positivity of this class is defined in [37]:

Definition 2. Let $\lambda$ be a function, then $\lambda$ belongs to the class of passive multipliers $\mathcal{P}$ if $\lambda(\omega)=\lambda(\omega)^{*}$ and $\lambda(\omega)>0$.

Lemma 3 ( [37]). Given a bounded LTI passive system $\Delta$ and $\lambda \in \mathcal{P}$, then $\Delta$ satisfies the IQC defined by

$$
\Pi(\omega)=\left[\begin{array}{cc}
0 & \lambda(\omega) \\
\lambda(\omega) & 0
\end{array}\right] .
$$

Definition 3 (Zames-Falb Multiplier [26], [27]). Let $Z$ be a rational transfer function. Then $Z$ belongs to the multiplier class of Zames-Falb multipliers $\mathcal{Z}$, if the following three conditions are satisfied:

$$
\begin{aligned}
& \text { - } Z(j \omega)=z_{0}-\int_{-\infty}^{\infty} q(t) e^{-j \omega t} d t, \\
& \text { - } \quad \int_{-\infty}^{\infty}|q(t)| d t<z_{0}, \\
& \text { - } \quad q(t) \geq 0, \quad \forall t \in \mathbb{R} .
\end{aligned}
$$

Lemma 4 ( [27]). Given a memoryless, monotone and bounded nonlinearity $\phi$ and any $Z \in \mathcal{Z}$, then the nonlinearity satisfies the IQC defined by

$$
\Pi(j \omega)=\left[\begin{array}{cc}
0 & Z(j \omega)^{*} \\
Z(j \omega) & 0
\end{array}\right] .
$$

A comparison between the rest of the classes for this type of nonlinearity and the class $\mathcal{Z}$ is given in [38].

In the IQC framework, the uncertainty based on delay operator is encapsulated with a negative unit, i.e. $\Delta_{d}(j \omega)=$ $e^{-j \omega T_{d}}-1$, see Fig. 5. Hence one can think of the block $\Delta_{d}$ as a perturbation of the feedback without delay.

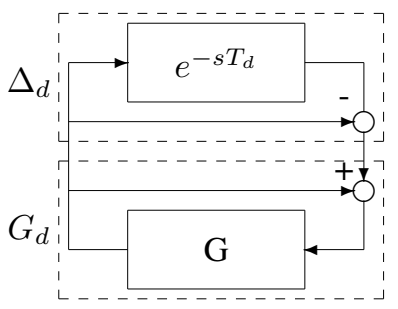

Fig. 5. Encapsulation of the time delay operator in a way to $\Delta_{d}=e^{-s T_{d}}-1$.

Definition 4. Given a delay $T_{d m a x}$, $\Pi$ belongs to the class of multipliers $\Pi_{d 1}$ if there exist $D(\omega)=D^{*}(\omega) \geq 0, \Omega(\omega)=$ $\Omega^{*}(\omega) \leq 0$, and $W_{d}(j \omega)$ ensures $\left|W_{d}(j \omega)\right| \geq\left|\Delta_{d}(j \omega)\right|$, $\forall T_{d} \in\left[0, T_{d \max }\right], \forall \omega \in \mathbb{R}$, such that

$$
\Pi(j \omega)=\left[\begin{array}{cc}
W_{d}(j \omega)^{*} D(\omega) W_{d}(j \omega) & \Omega(\omega) \\
\Omega(\omega) & -D(\omega)+\Omega(\omega)
\end{array}\right] .
$$

Remark 2. Generally, multiplier classes for time delay are defined with any $\Omega(\omega)$, yet the negativity condition $-D(\omega)+$ 
$\Omega(\omega)$ was included to be ensure that right lower part of the multiplier is negative definite. It is possible to relax this condition on $\Omega$ with $\Omega(\omega) \leq D(\omega)$ but it does not lead to useful results in this paper.

Lemma 5. Let $\Delta_{d}(j \omega)$ be a time delay operator with constant unknown time delay $T_{d} \in\left[0, T_{d \max }\right]$, it satisfies the IQC defined by $\Pi \in \Pi_{d 1}$.

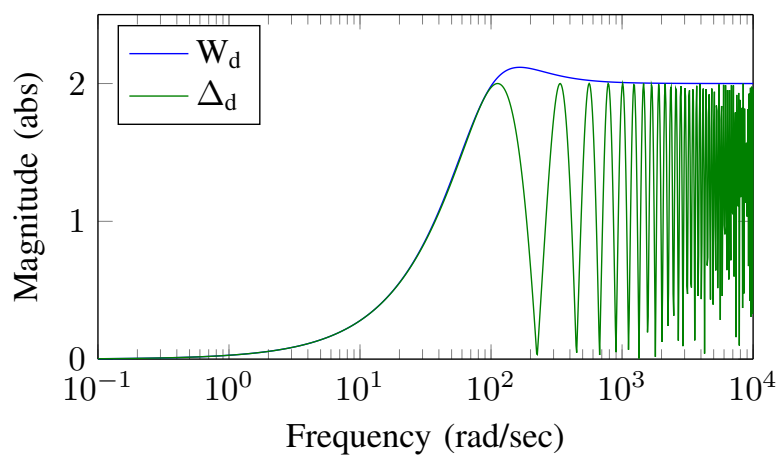

Fig. 6. Covering gain of the delay with a rational transfer function, $T_{d \max }=$ $28 \mathrm{msec}$.

Amplitude covering property of the $W_{d}$ can be seen in Fig.6, with following rational transfer function [39]

$$
W_{d}(j \omega)=2 \frac{(j \omega)^{2} T_{d \max }^{2}+3.5 j \omega T_{d \max }+10^{-6}}{(j \omega)^{2} T_{d \max }^{2}+4.5 j \omega T_{d \max }+7.1}
$$

Based on the behaviours of the $\Delta_{d}$ on the complex plane another IQC can be defined with rational approximation of the delay operator.

Definition 5. Given a delay $T_{d \max }$, $\Pi$ belongs to the class of multipliers $\boldsymbol{\Pi}_{d 2}$ if there exist $D(\omega)=D^{*}(\omega) \geq 0, \Omega(\omega)=$ $\Omega^{*}(\omega) \leq 0$, and $\Upsilon_{d}(j \omega)$ with $\Delta_{d} \in C\left(\Upsilon_{d},\left|\Upsilon_{d}\right|\right), \forall T_{d} \in$ $\left[0, T_{d \max }\right], \forall \omega \in \mathbb{R}$, such that

$\Pi(j \omega)=\left[\begin{array}{cc}0 & \Upsilon_{d}(j \omega)^{*} D(\omega)+\Omega(\omega) \\ \Upsilon_{d}(j \omega) D(\omega)+\Omega(\omega) & -D(\omega)+\Omega(\omega)\end{array}\right]$.

Lemma 6 ( [40]). Let $\Delta_{d}(j \omega)$ be a time delay operator with constant unknown time delay $T_{d} \in\left[0, T_{d \max }\right]$, then it satisfies the IQC defined by $\Pi \in \Pi_{d 2}$.

An example of $\Upsilon_{d}$ was defined in [34] as

$$
\Upsilon_{d}(j \omega)=\frac{-2.19(j \omega)^{2}+9.02\left(\frac{j \omega}{T_{d \max }}\right)+\frac{0.089}{T_{d \max }^{2}}}{(j \omega)^{2}-5.64\left(\frac{j \omega}{T_{d \max }}\right)-\frac{17}{T_{d \max }^{2}}} .
$$

Both multipliers can be used while analysing the uncertainties caused by constant time delays, but unfortunately there is no direct recipe to define which one gives better performance specifications.

Depending on the architecture of the system, delay may become time variable operator as well.

Definition 6. Given a variable delay $T_{d}(t) \in\left[0, T_{\text {dmax }}\right]$ with $\left|\dot{T}_{d}(t)\right| \leq d<1, \forall t \geq 0$, $\Pi$ belongs to the class of multipliers
$\Pi_{v}$ if there exist $D(\omega)=D^{*}(\omega) \geq 0, \Omega(\omega)=\Omega^{*}(\omega) \leq 0$, and $W_{v}(j \omega)$ is rational transfer function satisfying

$$
\left|W_{v}(j \omega)\right|> \begin{cases}1+\frac{1}{\sqrt{1-d}}, & \text { if } T_{d \max }|\omega|>1+\frac{1}{\sqrt{1-d}} \\ T_{d \max }|\omega|, & \text { if } T_{d \max }|\omega| \leq 1+\frac{1}{\sqrt{1-d}}\end{cases}
$$

then

$$
\Pi(j \omega)=\left[\begin{array}{cc}
W_{v}(j \omega) D W_{v}^{*}(j \omega)+\frac{d}{1-d} \Omega & \Omega \\
\Omega & -D+\Omega
\end{array}\right],
$$

where, $d$ is variation of the delay, $\dot{T}_{d}$.

Lemma 7 ( [41]). Let $\Delta_{d}$ be a variable time delay operator with delay $T_{d}(t) \in\left[0, T_{d \max }\right]$ and $\left|\dot{T}_{d}(t)\right| \leq d<1, \forall t \geq 0$, $\Delta_{d}$ satisfies $I Q C$ defined by $\Pi \in \Pi_{v}$.

\section{Parametrization And Combination of the MULTIPLIERS}

Following [23], given fixed positive integer $n$ and real $\alpha$ (see (1)), we will parametrize each multiplier using these two fixed values and an adequate matrix containing a set of parameters to carry out a convex search. We will describe all parametrizations using the same notation, though $n$ and $\alpha$ will be different for each multiplier when they are combined.

\section{A. Parametrizations}

Firstly, we use the same parametrization for frequency dependent passive multiplier $\lambda(\omega)$ as in [23]. Then a subclass of the set of multipliers $\mathcal{P}$ is given by $\lambda(\omega)=$ ${ }^{n} \Lambda_{\alpha}(j \omega)^{*} K_{h}{ }^{n} \Lambda_{\alpha}(j \omega)$, where $K_{h}=K_{h}^{\top}$, and $\lambda(\omega) \in \mathbb{R}$ under the condition $\lambda(\omega) \geq 0$. Henceforth we simplify the notation for passive multipliers by using $\Lambda_{h}(j \omega)={ }^{n} \Lambda_{\alpha}(j \omega)$ for some $n$ and $\alpha$.

Secondly, we will parametrize the Zames-Falb multiplier using Szegö's polynomials. Following [42] and [43], $Z \in \mathcal{Z}$ in Definition 3 can be approximated as

$$
Z(j \omega)=z_{0}-\sum_{i=1}^{n-1}\left(\frac{a_{i}}{(j \omega+\alpha)^{i}}-\frac{b_{i}(-1)^{i-1}}{(j \omega-\alpha)^{i}}\right),
$$

if $n$ is chosen sufficiently large. Initially we factorize class of Zames-Falb multiplier as $Z(j \omega)={ }^{n} \Lambda_{\alpha}(j \omega)^{*} K_{z}{ }^{n} \Lambda_{\alpha}(j \omega)$, where $\Lambda_{\alpha}$ is a basis function with the same structure as in (1), and $K_{z}$ is a matrix contains free parameters of the $Z$ function $\left(z_{0}, a_{i}\right.$ and $\left.b_{i}\right)$ at its first column and row

$$
K_{z}=\left[\begin{array}{ccccc}
z_{0} & -a_{1} & -a_{2} & \cdots & -a_{n-1} \\
-b_{1} & 0 & 0 & \cdots & 0 \\
-b_{2} & 0 & 0 & \cdots & 0 \\
\vdots & \vdots & \vdots & \ddots & \vdots \\
-b_{n-1} & 0 & 0 & \cdots & 0
\end{array}\right] .
$$

Condition (4) in Definition 3 requires the inverse two-sided Laplace transform of the summation in $(10)^{3}$. It is given by

$$
q(t)= \begin{cases}e^{-\alpha t}\left(a_{1}+t a_{2}+\cdots+\frac{t^{n-2} a_{n-1}}{(n-2) !}\right), & \text { if } t \geq 0 ; \\ e^{\alpha t}\left(b_{1}-t b_{2}+\cdots+(-1)^{n-2} \frac{t^{n-2} b_{n-1}}{(n-2) !}\right), & \text { if } t<0\end{cases}
$$

\footnotetext{
${ }^{3}$ The transfer function in (10) is noncausal, and its region of convergence (ROC) includes the imaginary axis. See Table 8.4 in [44].
} 
and using that $q(t) \geq 0$ for all $t$, it follows by direct integration that

$$
\int_{-\infty}^{\infty} q(t) d t=(a+b) \bar{\alpha}<z_{0}
$$

where $a$ and $b$ are vectors contain all free parameters as

$$
\begin{aligned}
a & =\left[\begin{array}{llll}
a_{1} & a_{2} & \cdots & a_{n-1}
\end{array}\right], \\
b & =\left[\begin{array}{llll}
b_{1} & b_{2} & \cdots & b_{n-1}
\end{array}\right],
\end{aligned}
$$

and $\bar{\alpha}$ is a vector designated as

$$
\bar{\alpha}=\left[\begin{array}{llll}
1 / \alpha & 1 / \alpha^{2} & \cdots & 1 / \alpha^{n-1}
\end{array}\right]^{\top} .
$$

The final constraint $q(t) \geq 0$ in Definition 3 is implemented as an LMI as follows. Firstly, two transfer functions are generated:

$$
\begin{aligned}
H_{1}(s)= & \frac{\sum_{i=1}^{n-1} a_{i}(-1)^{i-1} s^{2(i-1)}(i-1) !^{-1}}{(-s+1)^{n-1}(s+1)^{n-1}} \\
H_{2}(s)= & \frac{\sum_{i=1}^{n-1} b_{i}(-1)^{i-1} s^{2(i-1)}(i-1) !^{-1}}{(-s+1)^{n-1}(s+1)^{n-1}}
\end{aligned}
$$

then $q(t) \geq 0$ for all $t \in \mathbb{R}$ is equivalent to the conditions $H_{j}(j \omega) \geq 0$ for $j=1,2$ and all $\omega \in \mathbb{R}$; finally these two conditions are expressed as LMIs via KYP Lemma. For minimal state space representations of these transfer functions we refer reader to [32], [42], [43].

Remark 3. Note that the positivity of $q(t)$ given in (11) is equivalent to the positivity of the polynomials

$$
\left(a_{1}+t a_{2}+\frac{t^{2} a_{3}}{2 !}+\cdots+\frac{t^{n-2} a_{n-1}}{(n-2) !}\right),
$$

and

$$
\left(b_{1}+t b_{2}+\frac{t^{2} b_{3}}{2 !}+\cdots+\frac{t^{n-2} b_{n-1}}{(n-2) !}\right) \text {, }
$$

for all $t \geq 0$; since $e^{ \pm \alpha t}>0$ for all $t$. Then positivity of $q(t)$ is independent on the value of $\alpha$. By default, we check the positivity of $q(t)$ assuming $\alpha=1$. See the proof in [42] for further details. Similarly, same conclusion can be reached for the anticausal component of the multipliers.

Although this parametrization has been shown to be a complete parametrization when $N \rightarrow \infty$ [45], this limit is not feasible due to numerical issues. To increase the flexibility without increasing the order of the multiplier, we propose a second method similar to the parametrization proposed in [32], $Z(j \omega)=\Lambda_{\alpha, \beta}(j \omega)^{*} K_{e} \Lambda_{\alpha, \beta}(j \omega)$, so that flexibility of the asymmetric poles in a multiplier can be used (see example in [26], [28]). Let $\Lambda_{\alpha, \beta}(j \omega)$ be a second basis function given by

$$
\Lambda_{\alpha, \beta}(j \omega)=\left[{ }^{n} \Lambda_{\alpha}(j \omega)^{\top}{ }^{n} \Lambda_{\beta}(j \omega)^{\top}\right]^{\top}
$$

with $\beta, \alpha>0$, and $K_{e}$ containing free parameters of $Z$ function expressed as

$$
K_{e}=\left[\begin{array}{cccccccc}
z_{1} & -a_{1} & -a_{2} & \cdots & -a_{n-1} & 0 & \cdots & 0 \\
0 & 0 & 0 & \cdots & 0 & 0 & \cdots & 0 \\
\vdots & \vdots & \vdots & \ddots & \vdots & \vdots & \ddots & \vdots \\
0 & 0 & 0 & \cdots & 0 & z_{2} & \cdots & 0 \\
0 & 0 & 0 & \cdots & 0 & -b_{1} & \cdots & 0 \\
0 & 0 & 0 & \cdots & 0 & -b_{2} & \cdots & 0 \\
\vdots & \vdots & \vdots & \ddots & \vdots & \vdots & \ddots & \vdots \\
0 & 0 & 0 & \cdots & 0 & -b_{n-1} & \cdots & 0
\end{array}\right]
$$

and condition proposed in (12) is redefined as

$$
a \bar{\alpha}+b \bar{\beta}<z_{1}+z_{2},
$$

where $\bar{\beta}$ is defined similarly to $\bar{\alpha}$. Similarly, conditions on $H_{1}$ and $\mathrm{H}_{2}$ still ensure that the new $q(t)$ is non-negative for all $t \in \mathcal{R}$, as has been mentioned in Remark 3. Henceforward, we will use $\Lambda_{e}$ as the basis associated with the Zames-Falb multiplier, regardless of the selection on the poles and order. If one pole is used, it means that the first option has been used whereas the second option has been used if two poles are given.

Parametrization of the multipliers for uncertainties caused by time delay can be performed in a similar fashion as in passivity multiplier. For constant time delay case, infinite dimensional frequency dependent functions $\Omega(\omega)$ and positive definite $D(\omega)$ can be parametrized as

$$
\begin{aligned}
& D(\omega)={ }^{n} \Lambda_{\alpha}(j \omega)^{*} K_{d}{ }^{n} \Lambda_{\alpha}(j \omega), \\
& \Omega(\omega)={ }^{n} \Lambda_{\alpha}(j \omega)^{*} K_{r}{ }^{n} \Lambda_{\alpha}(j \omega),
\end{aligned}
$$

where $K_{d, r}=K_{d, r}^{T}$, and basis functions $\Lambda_{\alpha}$ are designed as in (1) with different poles and orders. Henceforward we will use the notation $\Lambda_{d}$ and $\Lambda_{r}$ to represent some selection for the poles and orders of these two multipliers.

For clarity, we parametrize and factorize $\Pi \in \Pi_{d_{2}}$ as follows

$$
\Pi=(\star)^{*}\left[\begin{array}{cccc}
0 & K_{d} & 0 & 0 \\
K_{d} & -K_{d} & 0 & 0 \\
0 & 0 & 0 & K_{r} \\
0 & 0 & K_{r} & K_{r}
\end{array}\right]\left[\begin{array}{cc}
\Lambda_{d} \Upsilon_{d} & 0 \\
0 & \Lambda_{d} \\
\Lambda_{r} & 0 \\
0 & \Lambda_{r}
\end{array}\right]
$$

where, dependence on $(j \omega)$ has been omitted.

It can be noted that IQC defined for time variable delays, (9), is equal to (7) when $\dot{T}_{d}(t)=0$ (constant delay case).

\section{B. Combination of The Classes of Multipliers}

We have already identified in Section II (Figs 2(b) and 3(b)) two important uncertain structures in bilateral teleoperation. However, a third class of structure will be analysed since it is relevant in other problems and our result can be benchmarked with other results in the literature. This class is a diagonal structure consisting of a slope-restricted nonlinearity and the time delay operator $\Delta_{d}$. In this section we generate the class of multipliers for those three structures depicted in Fig. 7. 


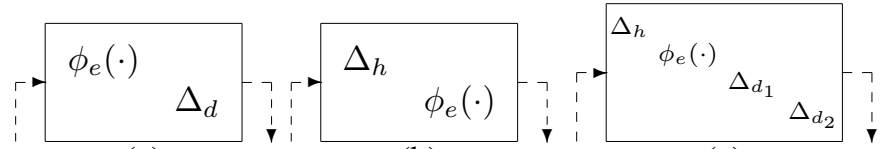

(a)

(b)

(c)

Fig. 7. Structured uncertainties: (a) is nonlinearity and time delay, (b) is passive plus nonlinear, and (c) is combination of the passive, nonlinear, and time delays.

Firstly, let us define the class of multipliers for the structure in Fig. 7 (a) by combining the class of Zames-Falb multipliers (6) and multipliers proposed for time delay (8).

Definition 7. Given some selection of $\Lambda_{e}, \Lambda_{d}$, and $\Lambda_{r}$, let $\Pi_{a}$ be the class of multipliers with the following structure:

$$
\Pi=(\star)^{*} \underbrace{\left[\begin{array}{cccccccc}
0 & 0 & K_{e}^{\top} & 0 & 0 & 0 & 0 & 0 \\
0 & 0 & 0 & K_{d} & 0 & 0 & 0 & 0 \\
K_{e} & 0 & 0 & 0 & 0 & 0 & 0 & 0 \\
0 & K_{d} & 0 & -K_{d} & 0 & 0 & 0 & 0 \\
0 & 0 & 0 & 0 & 0 & 0 & 0 & 0 \\
0 & 0 & 0 & 0 & 0 & 0 & 0 & K_{r} \\
0 & 0 & 0 & 0 & 0 & 0 & 0 & 0 \\
0 & 0 & 0 & 0 & 0 & K_{r} & 0 & K_{r}
\end{array}\right]}_{K_{a}} \underbrace{\left[\begin{array}{l}
\Psi_{a}(1,1) \\
\Psi_{a}(2,1)
\end{array}\right]}_{\Psi_{a}},
$$

where, $\Psi_{a}(1,1)=\operatorname{diag}\left(\Lambda_{e}, \Lambda_{d} \Upsilon_{d}, \Lambda_{e}, \Lambda_{d}\right)$, $\Psi_{a}(2,1)=\operatorname{diag}\left(0, \Lambda_{r}, 0, \Lambda_{r}\right)$, and $K_{e}, K_{d}, K_{r}$ are defined by (15), and (17), respectively. Then, $\Pi \in \Pi_{a}$ if the following constraint and LMIs hold if

$\bullet$

$$
a \bar{\alpha}+b \bar{\beta}<z_{1}+z_{2},
$$

- there exists $X_{j}=X_{j}^{\top}$ such that,

$$
\left[\begin{array}{cc}
A_{j}^{\top} X_{j}+X_{j} A_{j} & C_{j}^{\top}-X_{j} B_{j} \\
C_{j}-B_{j}^{\top} X_{j} & -\left(D_{j}+D_{j}^{\top}\right)
\end{array}\right] \leq 0,
$$

where $A_{j}, B_{j}, C_{j}, D_{j}$ are state space parameters of $H_{j}(s)$ in (13), for $j=1,2$,

- there exist symmetric matrices, $P_{d}, K_{d}$ such,

$\left[\begin{array}{cc}A_{d}^{\top} P_{d}+P_{d} A_{d} & P_{d} B_{d} \\ B_{d}^{\top} P_{d} & 0\end{array}\right]-(\star)^{\top} K_{d}\left[\begin{array}{cc}C_{d} & D_{d}\end{array}\right] \leq 0$,

- there exist symmetric matrices $P_{r}, K_{r}$ such that,

$$
\left[\begin{array}{cc}
A_{r}^{\top} P_{r}+P_{r} A_{r} & P_{r} B_{r} \\
B_{r}^{\top} P_{r} & 0
\end{array}\right]+(\star)^{\top} K_{r}\left[\begin{array}{cc}
C_{r} & D_{r}
\end{array}\right] \leq 0,
$$

where $A_{j}, B_{j}, C_{j}, D_{j}$ are state space parameters of the basis functions $\Lambda_{j}(j \omega)$, for $j=d$, r.

Lemma 8. Given an uncertainty $\Delta=\operatorname{diag}\left(\phi_{e}, \Delta_{d}\right)$ as in Fig. 7 (a) and $\Pi \in \Pi_{a}$, then $\Delta$ satisfies the IQC defined by $\Pi(j \omega)$.

Secondly, we develop the class of multipliers that we will use to analyse the absolute stability of the bilateral teleoperated system when the human block is modelled as an LTI passive system and the environment as a bounded and monotone nonlinearity, see Fig. 7 (b). We combine frequency dependent multipliers for LTI passive systems, (2), and Zames-Falb multipliers, (6) as follows:
Definition 8. Given some selection of $\Lambda_{h}$ and $\Lambda_{e}$, then let us consider the multiplier with the following structure:

$$
\Pi=(\star)^{*} \underbrace{\left[\begin{array}{cccc}
0 & 0 & K_{h} & 0 \\
0 & 0 & 0 & K_{e}^{\top} \\
K_{h} & 0 & 0 & 0 \\
0 & K_{e} & 0 & 0
\end{array}\right]}_{K_{b}}\left[\begin{array}{cc}
\Psi_{b}(j \omega) & 0 \\
0 & \Psi_{b}(j \omega)
\end{array}\right] \text {, }
$$

where $K_{h}$ is any symmetric matrix, $K_{e}$ is defined in (15), and $\Psi_{b}(j \omega)=\left[\begin{array}{cc}\Lambda_{h}(j \omega) & 0 \\ 0 & \Lambda_{e}(j \omega)\end{array}\right]$. Then, $\Pi \in \Pi_{b}$ if the following statements hold:

- there exist symmetric matrices $P_{h}$ and $K_{h}$ such that,

$\left[\begin{array}{cc}A_{h}^{\top} P_{h}+P_{h} A_{h} & P_{h} B_{h} \\ B_{h}^{\top} P_{h} & 0\end{array}\right]-(\star)^{\top} K_{h}\left[\begin{array}{cc}C_{h} & D_{h}\end{array}\right] \leq 0$,

where $A_{h}, B_{h}, C_{h}$, and $D_{h}$ are the state space representation of $\Lambda_{h}(j \omega)$,

- constraint (19) and LMI (20) are satisfied.

Lemma 9. Given a structured uncertainty block $\Delta=\operatorname{diag}\left(\Delta_{h}, \phi_{e}\right)$ as in Fig. $7(b)$, then $\Delta$ satisfies the IQC defined by $\Pi(j \omega) \in \Pi_{b}$.

Definition 9. Given some selection of $\Lambda_{h}, \Lambda_{e}, \Lambda_{d_{1}}, \Lambda_{d_{2}}, \Lambda_{r_{1}}$, $\Lambda_{r_{2}}$ and rational transfer functions $\Upsilon_{d_{i}}, i=1,2$; let $\boldsymbol{\Pi}_{c}$ be the class of multipliers with the following structure:

$$
\Pi=(\star)^{*} \underbrace{\left[\begin{array}{cccc}
0 & \bar{K}_{1}^{\top} & 0 & 0 \\
\bar{K}_{1} & \bar{K}_{2} & 0 & 0 \\
0 & 0 & 0 & \bar{K}_{3} \\
0 & 0 & \bar{K}_{3} & \bar{K}_{3}
\end{array}\right]}_{\bar{K}}\left[\begin{array}{l}
\Psi_{c}(1,1) \\
\Psi_{c}(2,1)
\end{array}\right],
$$

where $\bar{K}_{1}=\operatorname{diag}\left(K_{h}, K_{e}, K_{d_{1}}, K_{d_{2}}\right), \quad \bar{K}_{2}=$ $\operatorname{diag}\left(0,0,-K_{d_{1}},-K_{d_{2}}\right), \quad \bar{K}_{3}=\operatorname{diag}\left(0,0, K_{r_{1}}, K_{r_{2}}\right)$, and $\Psi_{c}(1,1)=\operatorname{diag}\left(\Lambda_{h}, \Lambda_{e}, \Lambda_{d_{1}} \Upsilon_{d_{1}}, \Lambda_{d_{2}} \Upsilon_{d_{2}}, \Lambda_{h}, \Lambda_{e}, \Lambda_{d_{1}}, \Lambda_{d_{2}}\right)$, $\Psi_{c}(2,1)=\operatorname{diag}\left(0,0, \Lambda_{r_{1}}, \Lambda_{r_{2}}, 0,0, \Lambda_{r_{1}}, \Lambda_{r_{2}}\right)$. Then $\Pi \in \Pi_{c}$ if constraint (19) and LMIs (20), (21), (22), and (24) are satisfied $^{4}$.

Lemma 10. A constructed uncertainty block $\Delta=\operatorname{diag}\left(\Delta_{h}, \phi_{e}, \Delta_{d_{1}}, \Delta_{d_{2}}\right)$, as in Fig. 7 (c), satisfies the IQC defined by $\Pi(j \omega) \in \Pi_{c}$.

\section{Main Results}

Initially, we use Lemma 8 to analyse system with saturation and time delay via IQC theorem, where the results can be compared with other techniques. Afterwards, we apply Lemmas 9 and 10 to bilateral teleoperation.

\section{A. Systems with Saturation and Time Delay}

Stability of time delay system has attracted much attention over the last decades. The literature is dominated by the Lyapunov-Krasovskii functional technique [46], but IQC framework has been also used [40], [41], [47], [48].

Time-domain techniques such as Lyapunov-Krasovskii functional are versatile tools for time-delay systems; but when delay is combined with other nonlinearities as saturation, the

${ }^{4}$ LMIs for $D_{1,2}(\omega)$ and $\Omega_{1,2}(\omega)$ need to be defined for each delay 
modularity of IQC framework may provide some advantages. For example, if the nonlinearity is slope or sector bounded then Zames-Falb multipliers outperform Lyapunov techniques [28], [38]. In this section, we focus our attention on the absolute stability of a system containing both slope-restricted nonlinearities and constant time delay in an interval $\left[0, T_{d \max }\right]$.

The system is transformed into feedback connection between nominal plant $G$ and structured uncertainty, see Fig. 8 . Then absolute stability of the whole system is transformed to a search for appropriate multipliers within the defined class such that $\Pi(j \omega) \in \Pi_{a}$.

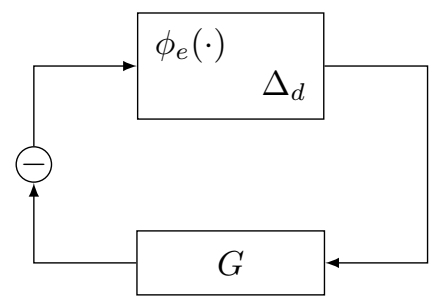

Fig. 8. Time delayed Lurye system, where $\Delta$ is structured uncertainty contains bounded nonlinearity, $\phi_{e}$, and encapsulated time delay, $\Delta_{d}$.

Corollary 11. Consider the negative feedback interconnection between $G$ and structured uncertainty block in Fig. 7 (a). Let us define a minimal state space representation as

$$
\Psi_{a}(j \omega)\left[\begin{array}{c}
-G(j \omega) \\
I
\end{array}\right] \sim\left[\begin{array}{ll}
A_{a} & B_{a} \\
C_{a} & D_{a}
\end{array}\right],
$$

where $\Psi_{a}(j \omega)$ is defined in (18). Assume that interconnection between $G$ and $\tau \Delta$ is well posed for $\tau \in[0,1]$. Then the interconnection is absolutely stable if there exist symmetric matrices $P_{a}$ and $K_{a}$; where $K_{a}$ is given in (18), such that there exists $\Pi=(\star)^{*} K_{a}\left[\begin{array}{cc}\Psi_{a}(j \omega) & 0 \\ 0 & \Psi_{a}(j \omega)\end{array}\right] \in \Pi_{a}$ and following LMI holds

$$
\left[\begin{array}{cc}
A_{a}^{\top} P_{a}+P_{a} A_{a} & P_{a} B_{a} \\
B_{a}^{\top} P_{a} & 0
\end{array}\right]+(\star)^{\top} K_{a}\left[\begin{array}{cc}
C_{a} & D_{a}
\end{array}\right] \leq 0 .
$$

Proof. If LMIs (20), (21), and (22) hold with defined conditions, then uncertainty block in Fig. 7 (a) satisfies IQC defined by $\Pi(j \omega) \in \boldsymbol{\Pi}_{a}$, see Lemma 8 .

Well-posedness condition in IQC theorem was assumed to be satisfied and as the lower right corner of $\Pi(j \omega) \in \Pi_{a}$ is negative semi-definite, $\tau \Delta$ satisfies the IQC defined by for $\tau \in[0,1]$ if $\Delta$ satisfies so second condition in the theorem is streamlined. Thirdly, based on the KYP Lemma, satisfying the LMI (26) implies that

$$
\left[\begin{array}{c}
-G(j \omega) \\
I
\end{array}\right]^{*} \Pi(j \omega)\left[\begin{array}{c}
-G(j \omega) \\
I
\end{array}\right] \leq-\epsilon I, \quad \forall \omega \in \mathbb{R},
$$

also holds for some $\epsilon>0$ and based on the Theorem 1 it can be concluded that the proposed interconnection is stable.

Eventually, the infinite dimensional inequality in the IQC theorem is converted into an LMI which is a convex cone and search can be carried out with semi definite programs.

\section{B. Teleoperation with Memoryless Monotonic Environment}

In this section, we develop results to analyse the stability of the bilateral teleoperation when human operator and environment are modelled as LTI passive system, and a monotone and bounded nonlinearity, respectively. Then, we need to use parametrization of both multipliers, $\lambda(\omega)$ and $Z(j \omega)$ in order to develop a convex search for proper class of multipliers such that results give idea about the stability condition of the interconnection depicted in Fig. 9.

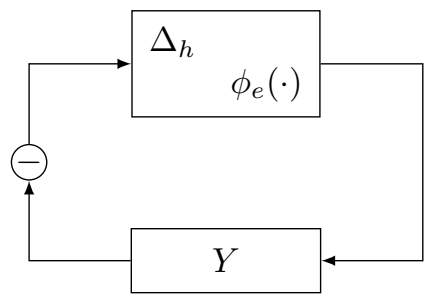

Fig. 9. Delay free bilateral teleoperation as a classical nominal plantuncertainty interconnection.

Corollary 12. Consider the negative feedback interconnection between admittance matrix $Y$ and structured uncertainty in Fig. 7 (b). Let us consider the following minimal state space representation:

$$
\left[\begin{array}{cc}
\Psi_{b}(j \omega) & 0 \\
0 & \Psi_{b}(j \omega)
\end{array}\right]\left[\begin{array}{c}
-Y(j \omega) \\
I
\end{array}\right] \sim\left[\begin{array}{cc}
A_{b} & B_{b} \\
C_{b} & D_{b}
\end{array}\right] .
$$

where $\Psi_{b}$ has been defined in (23). Assume that interconnection between $Y$ and $\tau \Delta$ is well posed for $\tau \in[0,1]$. Then, interconnection in Fig. 9 is absolutely stable if there exist symmetric matrices $P_{b}$ and $K_{b}$, where $K_{b}$ is given in (23), such that $(\star)^{*} K_{b}\left[\begin{array}{cc}\Psi_{b}(j \omega) & 0 \\ 0 & \Psi_{b}(j \omega)\end{array}\right] \in \boldsymbol{\Pi}_{b}$, and following LMI holds

$$
\left[\begin{array}{cc}
A_{b}^{\top} P_{b}+P_{b} A_{b} & P_{b} B_{b} \\
B_{b}^{\top} P_{b} & 0
\end{array}\right]+(\star)^{\top} K_{b}\left[\begin{array}{ll}
C_{b} & D_{b}
\end{array}\right] \leq 0 .
$$

Proof. If constraint (16) is satisfied with LMI (20), and LMI (24) holds, then there exists a multiplier class such that structured uncertainty satisfies IQC defined by $\Pi(j \omega) \in \Pi_{b}$, see Lemma 9. Finally, with KYP Lemma LMI (27) implies that,

$$
\left[\begin{array}{c}
-Y(j \omega) \\
I
\end{array}\right]^{*} \Pi(j \omega)\left[\begin{array}{c}
-Y(j \omega) \\
I
\end{array}\right] \leq-\epsilon I, \quad \forall \omega \in \mathbb{R}
$$

is satisfied for some $\epsilon>0$, where $Y(j \omega)$ is admittance transfer function matrix of the designed teleoperation system.

Remark 4. In [23] it has been stated that nominal system, $Y$, needs to be perturbed because the inequalities (28) cannot be satisfied when $\omega \rightarrow \infty$, as the plant is strictly proper. Here we use the same approach as [23]; using $Y+\zeta I$, with $\zeta=10^{-4}$, instead of $Y$. This constant is interpreted as having uncertainties within the sector $\left(0, \zeta^{-1}\right)$.

Additionally, delay is an inevitable phenomenon if master and slave manipulators are placed at different locations. Subsequently, to analyse absolute stability of the time delayed 
bilateral teleoperation, multipliers for passive, Zames-Falb, and time delay are required. Therefore, existence of any multiplier within the suitable class as in Definition 9 needs to be searched. Overall, the system is transformed into the interconnection of $\bar{Y}$ and uncertainty block in Fig. 7 (c), where $\bar{Y}(j \omega)$ is a transformation of the admittance matrix, correspondingly whole time delayed bilateral teleoperation is reconstructed as system illustrated in Fig. 10.

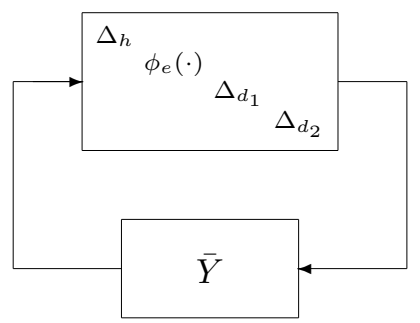

Fig. 10. Time delayed bilateral teleoperation as $\bar{Y}-\Delta$ interconnection, where human and environment are defined with $\Delta_{h}$ and $\phi_{e}$, respectively, and latencies between manipulators are characterized as $\Delta_{d i}=e^{-s T_{d i}}-1$, $i=(1,2)$.

Corollary 13. Consider feedback interconnection between $\bar{Y}$ and uncertainty block in Fig. 7 (c). Let us consider the following minimal state space representation:

$$
\Psi_{c}(j \omega)\left[\begin{array}{c}
\bar{Y}(j \omega) \\
I
\end{array}\right] \sim\left[\begin{array}{ll}
A_{c} & B_{c} \\
C_{c} & D_{c}
\end{array}\right]
$$

where $\Psi_{c}$ has been defined in (25). Assume that feedback interconnection between $\bar{Y}$ and $\tau \Delta$ is well posed for $\tau \in[0,1]$. Then, the feedback between $\bar{Y}$ and $\operatorname{diag}\left(\Delta_{h}, \phi_{e}, \Delta_{d 1}, \Delta_{d 2}\right)$ in Fig. 10 is stable if there exist symmetric matrices $P_{c}$ and $\bar{K}$; where $\bar{K}$ is given in (25), such that $\Psi_{c}^{*}(j \omega) \bar{K} \Psi_{c}(j \omega) \in \Pi_{c}$ and subsequent LMI holds

$$
\left[\begin{array}{cc}
A_{c}^{\top} P_{c}+P_{c} A_{c} & P_{c} B_{c} \\
B_{c}^{\top} P_{c} & 0
\end{array}\right]+(\star)^{\top} \bar{K}\left[\begin{array}{ll}
C_{c} & D_{c}
\end{array}\right] \leq 0
$$

Proof. If constraint (16) is satisfied with LMI (20), and LMIs (21), (22), (24) hold, then there exists a multiplier class such that structured uncertainty satisfies IQC defined by $\Pi(j \omega) \in \Pi_{c}$, see Lemma 10. In the end, based on the KYP Lemma, LMI (29) implies that

$\left[\begin{array}{c}\bar{Y}(j \omega) \\ I\end{array}\right]^{*} \Psi_{c}^{*}(j \omega) \bar{K} \Psi_{c}(j \omega)\left[\begin{array}{c}\bar{Y}(j \omega) \\ I\end{array}\right] \leq-\epsilon I, \quad \forall \omega \in \mathbb{R}$,

is satisfied for some $\epsilon>0$ and with IQC theorem it can be concluded that depicted interconnection in Fig. 10 is stable.

\section{EXAMPLES}

In this section, we show numerical examples of our main results, which are given as a convex optimization problem. We have used Yalmip with sdpt3 solver [49] to test the LMI conditions.

\section{A. Absolute Stability of Time Delayed System with Saturation}

In the first instance, validity of the proposed method was evaluated using an example which is commonly used in the literature [50]-[53]; an illustration of a system with slope restricted nonlinearity and delayed states. Consider the system given by

$$
\begin{aligned}
\dot{x}(t) & =A x(t)+B x\left(t-T_{d}\right)+D w(t), \\
m(t) & =M x(t)+N x\left(t-T_{d}\right), \\
w(t) & =-\phi(t, m(t)),
\end{aligned}
$$

where $\phi(t, m(t))$ belongs to the slope-restricted ${ }^{5}\left[K_{1}, K_{2}\right]$, $x(t) \in \mathcal{R}^{n}, w(t) \in \mathcal{R}^{k}, m(t) \in \mathcal{R}^{k}$ are the state, input, and output of the system, respectively. Let $A \in \mathcal{R}^{n \times n}, B \in \mathcal{R}^{n \times n}$, $D \in \mathcal{R}^{n \times k}, M \in \mathcal{R}^{k \times n}$, and $N \in \mathcal{R}^{k \times n}$ be known constant matrices as

$$
\begin{array}{rr}
A=\left[\begin{array}{cc}
-2 & 0 \\
0 & -0.9
\end{array}\right], \quad B=\left[\begin{array}{cc}
-1 & 0 \\
-1 & -1
\end{array}\right] \\
D=\left[\begin{array}{l}
-0.2 \\
-0.3
\end{array}\right], \quad M=\left[\begin{array}{ll}
0.6 & 0.8
\end{array}\right] \\
N=\left[\begin{array}{ll}
0 & 0
\end{array}\right], \quad K_{1}=0.2, K_{2}=0.5
\end{array}
$$

The system (30) is transformed into the interconnection depicted in Fig. 8. The stability of the system with the parameters given in (31) was transformed into a convex search for multiplier class in Definition 7, and results were obtained by using Corollary 11. For comparison and evaluation of the method, results are given in Table I.

TABLE I

MAXIMUM ALLOWABLE TIME DELAYS IN SYSTEM WITH SLOPE RESTRICTED NONLINEARITY

\begin{tabular}{|l|c|}
\hline Method & Maximum delay, $T_{d \max }$ \\
\hline Han [50] & 2.4859 \\
\hline Wu et al. $[51](\mathrm{n}=2)$ & 3.0080 \\
\hline Wu et al. $[51](\mathrm{n}=3)$ & 3.1110 \\
\hline Kazemy and Farrokhi [52] & 3.0216 \\
\hline Zeng et al. $[53](k=4)$ & 3.1730 \\
\hline $\boldsymbol{\Pi}_{a}$ (i.e. using $\left.\boldsymbol{\Pi}_{d 2}\right)$ & $\mathbf{3 . 2 1 9 1}$ \\
\hline Counterpart of $\boldsymbol{\Pi}_{a}$ using $\mathbf{\Pi}_{d 1}$ & $\mathbf{3 . 2 3 7 8}$ \\
\hline Nyquist value $($ only for $\phi(u)=k u)$ & 3.2520 \\
\hline
\end{tabular}

The proposed method leads to the less conservative results under the mild assumption of slope-restriction; maximum time delay for which we can guarantee the stability of the system with any nonlinearity within the defined class. These results were obtained with the proposed parametrization and combination of the multipliers, values of the selected parameters are given in Table II. A linear search was carried out to gain the basis function parameters and for this particular example it was observed that they do not have significant effect on the final results. Optimal design of basis functions is still an open question, and its selection depends on designer's ability. For Zames-Falb multipliers, automatic selection of the poles has been proposed in [38], [55], but their techniques can not be implemented here since they require odd nonlinearities.

\footnotetext{
${ }^{5}$ Previous results in literature have only considered sector conditions, but slope-restriction is a standard assumption in constrained input control [54] or delayed neural network [30], [33]
} 
Meanwhile, similar results were obtained while using asymmetric poles in multiplier with (14) as in both cases positivity of the nominal system was recovered, see Fig. 11. Orders and poles of the basis functions $\Lambda_{d}$ and $\Lambda_{r}{ }^{6}$ will remain same for the rest of analyses in this paper.

TABLE II

BASIS FUNCTIONS' PARAMETERS FOR RETARDED SYSTEM WITH NONLINEARITY

\begin{tabular}{|c|c|c|c|c|c|}
\cline { 2 - 6 } \multicolumn{1}{c|}{} & $\Lambda_{e}$ & $\Lambda_{d_{1}}$ & $\Lambda_{r_{1}}$ & $\Lambda_{d_{2}}$ & $\Lambda_{r_{2}}$ \\
\hline Order $(n)$ & 5 & 4 & 4 & 4 & 4 \\
\hline Pole $(-\alpha)$ & -10 & -21 & -14 & -13 & -8 \\
\hline
\end{tabular}

These results establish the usefulness of the Zames-Falb and delay multipliers while interconnected system contains both monotonic nonlinearity and latency. Henceforth, we implement the methodology in the stability analyses of the bilateral teleoperation where nonlinearity and time delay naturally occur in environment and communication medium, respectively.

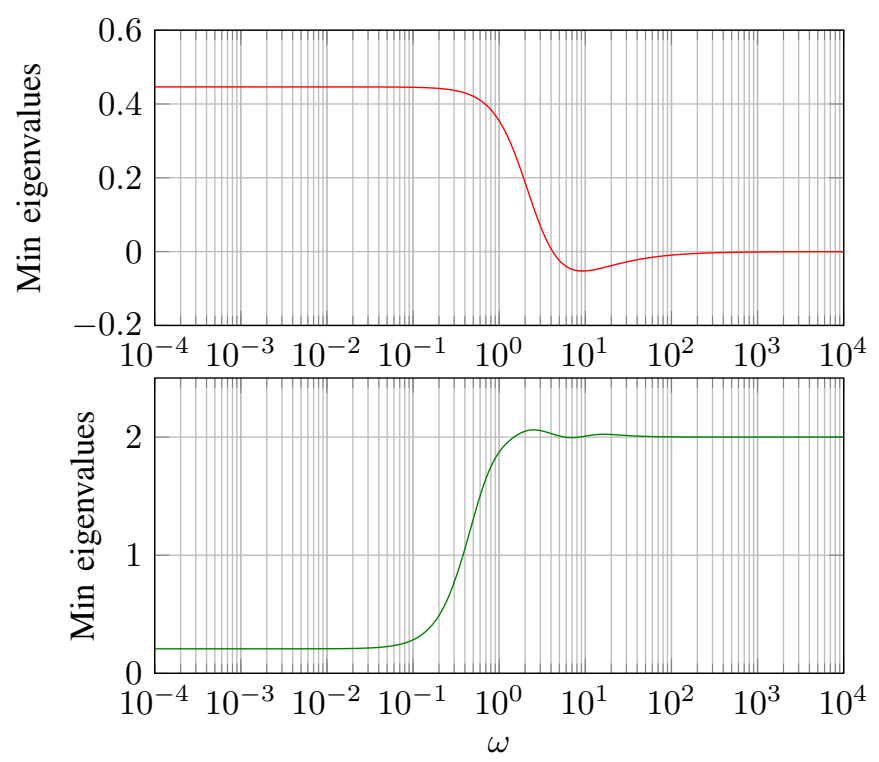

Fig. 11. Minimum eigenvalues of $G(j \omega)+G^{*}(j \omega)$ (Top): Nominal system is not positive definite without multiplier. Minimum eigenvalues with multiplier that ensures stability against $T_{d \max } \approx 3.2191 \mathrm{sec}$ (bottom).

\section{B. Stability of Bilateral Teleoperation}

The analyses of bilateral teleoperation are based on the position-force control architecture where master and slave are 1 degree-of-freedom (DOF) rigid robotic manipulators. System's equation of motions are

$$
v_{m}=Y_{m}\left(F_{h}+\tau_{m}\right), \quad v_{s}=Y_{s}\left(\tau_{s}-F_{e}\right),
$$

where $Y_{m}$ and $Y_{s}$ are the admittances of the manipulators, $\tau_{m}$ and $\tau_{s}$ are forces generated by controllers, $F_{h}$ and $F_{e}$ are applied human and environmental contact forces, respectively. Due to the rigid body conditions, manipulators' speeds are

${ }^{6}$ Number of the delay operators based on the system's dimension. equal to operator's and environment's speed such that $v_{m}=v_{h}$ and $v_{s}=v_{e}$. And controllers' forces are given by

$$
\begin{aligned}
\tau_{m} & =-K_{f} F_{e}, \\
\tau_{s} & =C_{s}\left(\mu x_{m}-x_{s}\right),
\end{aligned}
$$

where $C_{s}$ is the controller at the slave side and it is used for motion tracking, $K_{f}$ and $\mu$ are environmental interaction force and position scaling factors, respectively, $x_{m}$ and $x_{s}$ are manipulators' positions.

Based on this architecture different types of controllers $\left(C_{s}\right)$ can be implemented, yet it is generally designed as a PDcontroller such that $C_{s}(s)=K_{p}+K_{v} s$. P controller have also been implemented, but extra damping was inserted to certify stability

$$
\tau_{s}=K_{p}\left(\mu x_{m}-x_{s}\right)-K_{v} v_{s} .
$$

The values of the system parameters, which are used in the analyses, are given in Table III [14]. The parameters appertain to linearised 1 DOF Phantom Omni haptic manipulator, which was used in experiments, and controller's values are acquired with Internal Model Control (IMC) principles [56].

TABLE III

SYSTEM'S PARAMETERS

\begin{tabular}{|l|l|l|}
\hline \multicolumn{2}{|c|}{ Model } & Controller \\
\hline$M_{m}=0.001 \mathrm{~kg}$ & $M_{s}=0.001 \mathrm{~kg}$ & $K_{p}=10 \mathrm{~N} / \mathrm{m}$ \\
\hline$B_{m}=0.02 \mathrm{Ns} / \mathrm{m}$ & $B_{s}=0.02 \mathrm{~N} \mathrm{~s} / \mathrm{m}$ & $K_{v}=0.18 \mathrm{~N} \mathrm{~s} / \mathrm{m}$ \\
\hline
\end{tabular}

Two types of control algorithms are going to be tested with delayed and delay free bilateral teleoperation systems.

1) Delay Free Case: In some instances, master and slave manipulators are located close to each other such as surgery robotics where patient and surgeon are in the same room. Initially, we assumed that there is no latency in the communication medium. Let $Y_{m}(s)=\left(M_{m} s+B_{m}\right)^{-1}$ and $P_{p}(s)=\left(s^{2} M_{s}+s\left(B_{s}+K_{v}\right)+K_{p}\right)^{-1}$, the admittance matrix representation of damping injected, controlled with (32) and (34), P-F controller is

$$
Y_{P}(s)=\left[\begin{array}{cc}
Y_{m} & -K_{f} Y_{m} \\
-K_{p} \mu Y_{m} P_{p} & \left(K_{p} \mu Y_{m} K_{f}+s\right) P_{p}
\end{array}\right] .
$$

The system controlled with (32) and (33) will be called as PD-F architecture, and its admittance matrix representation is slightly different such that

$$
Y_{P D}(s)=\left[\begin{array}{cc}
Y_{m} & -K_{f} Y_{m} \\
-\mu\left(s K_{v}+K_{p}\right) Y_{m} P_{p} & Z_{22} Y_{m} P_{p}
\end{array}\right],
$$

where $Z_{22}(s)=s^{2} M_{m}+s\left(B_{m}+\mu K_{f} K_{v}\right)+\mu K_{f} K_{p}$. These admittance matrices are used within FDI, such as (28). As previously mentioned, stability is necessary but not the only criterion that needs to be considered while designing the bilateral teleoperation parameters.

Firstly, the absolute stability of the P-F control architecture was analysed. In the first stage Llewellyn's stability criterion was used under LTI passive operators assumption for both human and environment. The parameters are given in Table III, and the admittance matrix is given in (35). For the analysis, we choose the frequency range to be as $0 \mathrm{rad} / \mathrm{s}$ to 
TABLE IV

MAXIMUM OBTAINABLE TRANSPARENCY INDEXES

\begin{tabular}{|c|c|c|}
\cline { 2 - 3 } \multicolumn{1}{c|}{} & \multicolumn{2}{c|}{ Controller } \\
\cline { 2 - 3 } \multicolumn{1}{c|}{} & P-F Architecture & PD-F Architecture \\
\hline Passivity & 0.399 & - \\
\hline With symmetric poles & $\mathbf{0 . 6 4 5}$ & $\mathbf{0 . 4 1 8}$ \\
\hline With asymmetric poles & $\mathbf{0 . 8 0 9}$ & $\mathbf{0 . 6 2 2}$ \\
\hline
\end{tabular}

$1 \times 10^{6} \mathrm{rad} / \mathrm{s}$ where the maximum achievable transparency index is searched without destroying passivity of the 2-port network via bisection algorithm. It was concluded that with this hypothesis maximum achievable $K_{f} \mu$ value is approximately 0.399 so that admittance matrix is on the boundary of the positive realness. If the environment is also monotone and bounded, less conservative results were obtained with Zames-Falb multipliers by using Corollary 12; results are given in Table IV. If we use symmetric poles, we obtain 0.645 , whereas the result reaches 0.809 when asymmetric poles are considered.

Secondly, we have analysed stability of the bilateral teleoperation with PD-F controller architecture whose admittance matrix defined in (36) . As it is highlighted in [14] it is not possible to fulfil Llewellyn's stability criteria with this controller unless $M_{s}=0$. Namely the admittance matrix is not positive real, so it cannot be ensured that the design is absolutely stable with the parameters in Table III, when both human and environment are assumed to be passive LTI systems. However, with the novel assumption on the environment, the use of Zames-Falb multipliers in Corollary 12 allows us to conclude that PD-F controller architecture is absolutely stable and maximum achievable transparency index is 0.418 . Similarly, the transparency index can be improved by using asymmetric poles, reaching 0.622 . These results are obtained with parameters given in Table V.

TABLE V

Basis Functions' Parameters For Bilateral Teleoperation

\begin{tabular}{|c|c|c|c|c|c|c|}
\cline { 2 - 7 } \multicolumn{1}{c|}{} & \multicolumn{3}{c|}{ P-F Architecture } & \multicolumn{3}{c|}{ PD-F Architecture } \\
\cline { 2 - 7 } \multicolumn{1}{c|}{} & $\Lambda_{h}$ & \multicolumn{2}{c|}{$\Lambda_{e}$} & $\Lambda_{h}$ & \multicolumn{3}{c|}{$\Lambda_{e}$} \\
\hline Order $(n)$ & 5 & 6 & 6 & 5 & 6 & 6 \\
\hline Pole $(-\alpha)$ & -110 & -75 & $-500,-0.1$ & -110 & -380 & $-400,-0.01$ \\
\hline
\end{tabular}

2) Time Delayed Case: Admittance matrix representations of architectures are need to be redefined if there exist time delay in the communication medium; for instance with a constant delay P-F's admittance matrix become

$$
Y_{P d}(s)=\left[\begin{array}{cc}
Y_{m} & -K_{f} Y_{m} e^{-s T_{d}} \\
-K_{p} \mu Y_{m} P_{p} e^{-s T_{d}} & \left(K_{p} \mu Y_{m} K_{f} e^{-2 s T_{d}}+s\right) P_{p}
\end{array}\right]
$$

For simplicity, initially it was assumed that delay duration, $T_{d}$ is equal in both directions and then delay is pulled out from the admittance matrix to treat as a perturbation

$$
Y_{d}(s)=\left[\begin{array}{cccc}
Y_{m} & 0 & -Y_{m} & 0 \\
0 & \frac{s Y_{s}}{s+Y_{S} C_{s}} & 0 & \frac{Y_{s} C_{s}}{s+Y_{s} C_{s}} \\
0 & K_{f} & 0 & 0 \\
-\mu Y_{m} & 0 & \mu Y_{m} & 0
\end{array}\right]
$$

where $Y_{s}(s)=\left(M_{s} s+B_{s}\right)^{-1}$ and $Y_{d}(s)$ is interconnected with structured uncertainty block, $\Delta=\operatorname{diag}\left(\Delta_{h}, \phi_{e}, e^{-s T_{d}}, e^{-s T_{d}}\right)$.

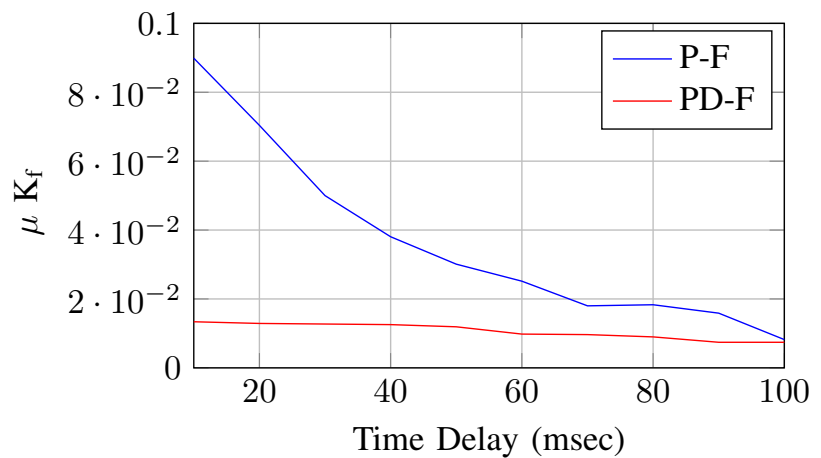

Fig. 12. Transparency index reduction against maximum delay duration in the communication medium.

In order to get the interconnection as illustrated in Fig. 10 first two channels of $Y_{d}$ need to be multiplied with negative sign, and due to the feed-forward inclusion in the delay channels positive feedbacks are included to the last two ports of the transfer function matrix, in that way $Y_{d}$ is transformed to $\bar{Y}$.

Initial studies have shown that having light and fast manipulators does largely degrade the stability margin when there exists time delay in the communication. With the mass and damping values given in Table III, performance index leads to small values so the scaled force signal might not be perceivable by human. In order to obtain reasonable performance indexes in delayed teleoperation, additional damping has virtually been injected into the master manipulator. Thus $B_{m}=0.2 \mathrm{~N} \mathrm{~s} / \mathrm{m}$ is used in the following analyses parameters. Test with several delay duration, $T_{d \max } \in[0.01,0.1] \mathrm{s}$, was carried out with bisection algorithm and maximum performance indexes for both type of controller are shown in Fig. 12. Basis function parameters in Table V were used with Corollary 13. For ZamesFalb multiplier the selection of the poles is very important, but for the delay we can reach same results with different poles.

For variable time delay, on the other hand, we have analysed characteristic of the internet communication medium between two laboratories; one way maximum transmission delay is approximately $28 \mathrm{msec}$ and variation of the delay is less than $0.45, \dot{T}_{d} \leq 0.45$. Based on these features bisection algorithm was carried out for searching maximum performance indexes and it was stated that with P-F controller $\mu K_{f}=0.0744$ and with PD-F architecture $\mu K_{f}=0.0261$, when $n=5$ in both controllers while using multipliers given in (2), (6), and (9) for uncertain operators human, environment, and time delay, respectively. As a result, these performance indexes are going to be used in the experimental evaluations.

3) Experimental Results: An experimental set-up is constructed between Manchester (UK) and Vigo (Spain) Universities and evaluation of the numerical results has been carried out experimentally with Phantom Omni haptic manipulators which have 6 DOF (3 actuated and 3 non-actuated joints). As analyses are not based on multi-DOF, only 1 DOF has been used while moving first joint and blocking/immobilizing the remaining. Omni manipulators have only position sensors so master's and slave's positions are sensed in radians with sensor located at the first joints, yet human and environmental 
forces are simulated with pre-designed passive and nonlinear operators, respectively.

Delay-free and constant time delay scenarios were carried out in the same laboratory while Omni manipulators are connected to a PC, where controllers are embedded into the Matlab/Simulink environment, through fire-wire cables and constant time delays are virtually injected to the communication medium. Variable time delay case, however, was carried out in two different laboratories; master and slave were located at The University of Manchester and University of Vigo, respectively. The internet was used as a communication medium and the data (position and force) were carried out with $\mathrm{UDP}^{7}$ packets. To be consistent in all cases, end effector of the master was aimed to follow hemicycle shape trajectory, meanwhile monotonic nonlinear type environmental force; $F_{e}\left(v_{s}\right)=k \arctan \left(v_{s}\right), k>0$, were acting on the slave. Using the designed controllers, the reference signal for the joint and master-slave manipulator behaviours as shown in Fig. 13.

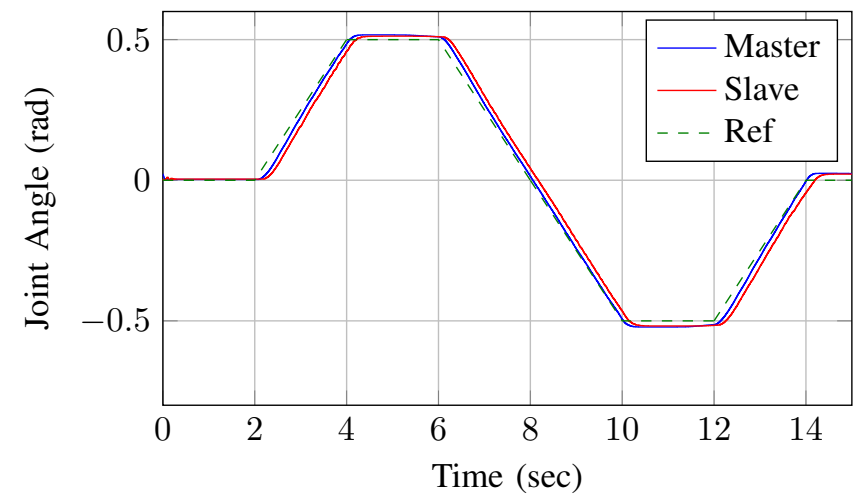

Fig. 13. Joint positions and reference signals; experiment taken between Manchester and Vigo.

Slave needs to mimic the behaviour of the master manipulator to be able to complete challenging tasks in an high quality manner. In order to evaluate this, we have tested the designed two control architectures, P-F and PD-F, with aforementioned maximum achievable transparency indexes. It is worth to highlight that P-F architecture position error is approximately 1.5 times higher than the PD-F architecture position in all scenarios, see Fig. 14. Fig. 15 shows that the improvement in the position tracking is not due to a reduction in the transparency, as PD-F architecture provides similar force mismatches as the P-F architecture under delay in the communication. As a result, Figures 14 and 15 demonstrate the benefit of the current analysis.

\section{CONCLUSION}

In this paper, we have analysed the stability of Lurye systems with memoryless, monotone, and bounded nonlinearities and time delays within their internal structure. The suggested methodology was evaluated with a commonly proposed system with saturation type nonlinearity plus time delay and results are competitive in comparison with results previously given in

\footnotetext{
${ }^{7}$ User Datagram Protocol (UDP) is one of the main internet communication protocols.
}
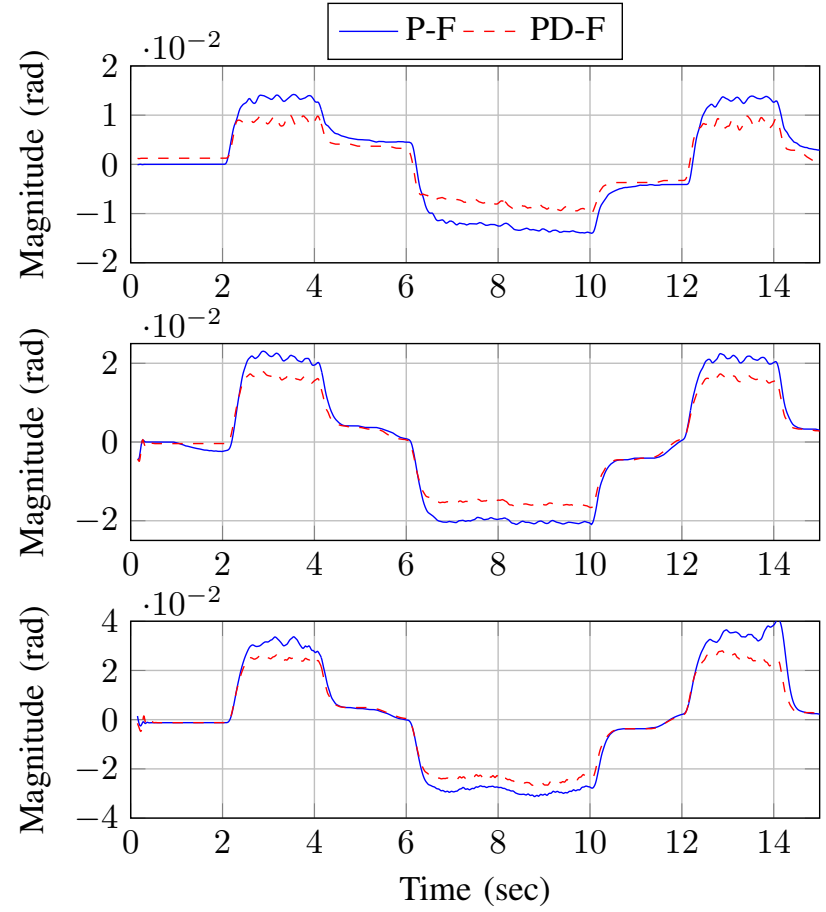

Fig. 14. Position mismatches between master and slave: Top is delay free case, middle is simulated constant delay (in one way $T_{d \max }=28 \mathrm{msec}$ ) between manipulators, and bottom is the experiment taken between Manchester and Vigo.

the literature. In particular, absolute stability of bilateral teleoperation was analysed by using passive multipliers, ZamesFalb multipliers, and suitable IQC descriptions for both timeinvariant and time-variant time delays. Parametrizations of these classes of multipliers allow us to transform the infinite dimensional problem into a convex optimization search over a subclass of multipliers.

We have demonstrated that the new description of the environments in conjunction with the IQC framework leads to less conservative conditions. As a result, we are able to achieve more transparent designs for bilateral teleoperation. The usefulness of this description has been demonstrated in a 2-channel architecture, where the term $Y_{22}$ in the admittance matrix is no longer restricted to be passive, since the Zames-Falb multiplier can correct some lack of passivity of this term. Moreover, the analysis has been validated with the experimental test bed between two distant laboratories with a communication over the internet. Both numerical and experimental evaluations show that the better description of the environment leads to less conservative results, yet with some computational load. Current results depend on user's ability to select the basis function for the parametrizations; hence further work is required to develop better selection of the poles and dimensions of the multiplier parametrizations.

\section{ACKNOWLEDGMENT}

The authors would like to thank to İlhan Polat and William Heath for the fruitful discussions and for revising early manuscript of the paper also to the anonymous reviewers for 

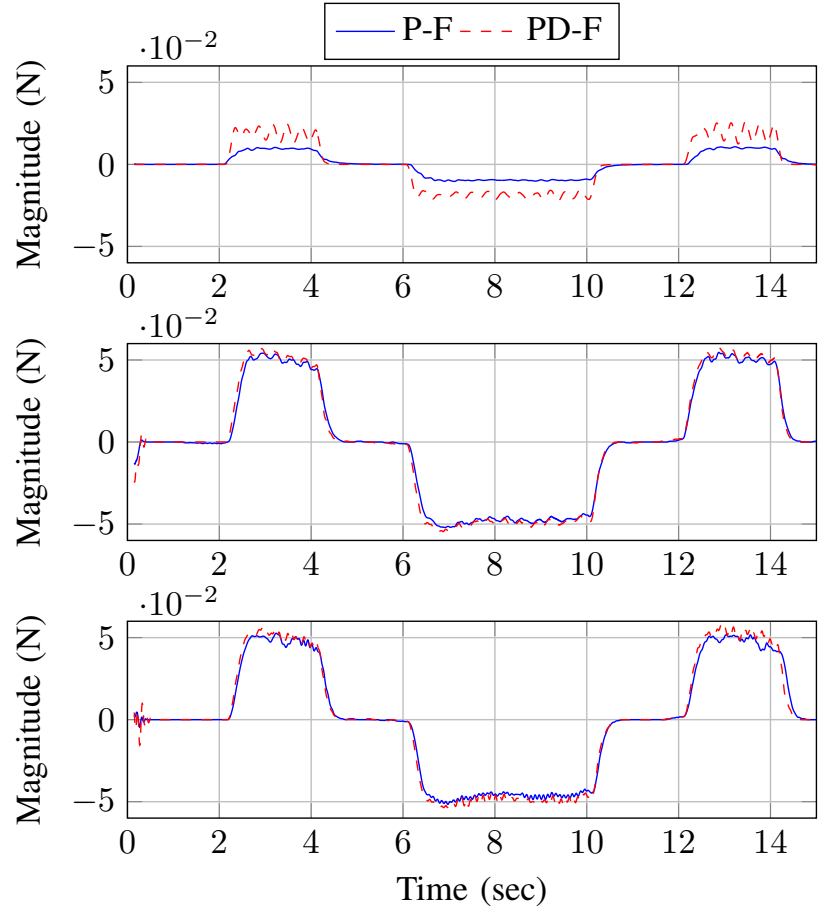

Fig. 15. Difference between the actual and transmitted (to master side) environmental interaction forces: Top is delay free case, middle is simulated constant delay (in one way $T_{d \max }=28 \mathrm{msec}$ ) between manipulators, and bottom is the experiment taken between Manchester and Vigo.

their helpful comments. This work has been partially funded by Turkish Ministry of Education.

\section{REFERENCES}

[1] J. Marescaux, J. Leroy, M. Gagner, F. Rubino, D. Mutter, M. Vix, S. E. Butner, and M. K. Smith, "Transatlantic robot-assisted telesurgery," Nature, vol. 413, no. 6854, pp. 379-380, 2001.

[2] X. Wang, W. Xu, B. Liang, and C. Li, "General scheme of teleoperation for space robot," in IEEE/ASME International Conference on Advanced Intelligent Mechatronics, jul 2008, pp. 341-346.

[3] J. De Geeter, M. Decréton, and E. Colon, "The challenges of telerobotics in a nuclear environment," Robotics and Autonomous Systems, vol. 28, no. 1 , pp. 5-17, jul 1999 .

[4] D. A. Lawrence, "Stability and transparency in bilateral teleoperation," IEEE Transactions on Robotics and Automation, vol. 9, no. 5, pp. 624637, 1993.

[5] P. F. Hokayem and M. W. Spong, "Bilateral teleoperation: An historical survey," Automatica, vol. 42, no. 12, pp. 2035-2057, dec 2006.

[6] E. Nuño, L. Basañez, and R. Ortega, "Passivity-based control for bilateral teleoperation: A tutorial," Automatica, vol. 47, no. 3, pp. 485495, mar 2011.

[7] A. Villaverde, A. Blas, J. Carrasco, and A. Torrico, "Reset control for passive bilateral teleoperation," IEEE Transactions on Industrial Electronics, vol. 58, no. 7, pp. 3037-3045, 2011.

[8] T. Hatanaka, Y. Igarashi, M. Fujita, and M. W. Spong, "Passivitybased pose synchronization in three dimensions," IEEE Transactions on Automatic Control, vol. 57, no. 2, pp. 360-375, 2012.

[9] C. A. Desoer and M. Vidyasagar, Feedback systems: input-output properties. SIAM, 1975, vol. 55 .

[10] F. B. Llewellyn, "Some fundamental properties of transmission systems," Proceedings of the IRE, vol. 40, no. 3, pp. 271-283, 1952.

[11] M. Tavakoli, A. Aziminejad, R. V. Patel, and M. Moallem, "Enhanced transparency in haptics-based master-slave systems," in American Control Conference, ACC '07, jul 2007, pp. 1455-1460.

[12] A. Jafari and J.-H. Ryu, "Input-to-state stable approach to release the conservatism of passivity-based stable haptic interaction," in International Conference on Robotics and Automation (ICRA). IEEE, 2015, pp. 285-290.
[13] A. Haddadi and K. Hashtrudi-Zaad, "Bounded-impedance absolute stability of bilateral teleoperation control systems," IEEE Transactions on Haptics, vol. 3, no. 1, pp. 15-27, jan 2010.

[14] B. Willaert, B. Corteville, D. Reynaerts, H. Van Brussel, and E. B. Vander Poorten, "A mechatronic analysis of the classical position-force controller based on bounded environment passivity," The International Journal of Robotics Research, vol. 30, no. 4, pp. 444-462, aug 2010.

[15] R. Anderson and M. Spong, "Bilateral control of teleoperators with time delay," IEEE Transactions on Automatic Control, vol. 34, no. 5, pp. 494-501, may 1989.

[16] G. Niemeyer and J.-J. E. Slotine, "Stable adaptive teleoperation," IEEE Journal of Oceanic Engineering, vol. 16, no. 1, pp. 152-162, 1991.

[17] _ "Using wave variables for system analysis and robot control," in International Conference on Robotics and Automation, vol. 2. IEEE, 1997, pp. 1619-1625.

[18] A. Eusebi and C. Melchiorri, "Force reflecting telemanipulators with time-delay: Stability analysis and control design," IEEE Transactions on Robotics and Automation, vol. 14, no. 4, pp. 635-640, 1998.

[19] N. Chopra, M. W. Spong, R. Ortega, and N. E. Barabanov, "On tracking performance in bilateral teleoperation," IEEE Transactions on Robotics, vol. 22, no. 4, pp. 861-866, aug 2006.

[20] T. Hatanaka, N. Chopra, M. Fujita, and M. W. Spong, Passivity-Based Control and Estimation in Networked Robotics. Springer, 2015.

[21] D. Lee and M. W. Spong, "Passive bilateral teleoperation with constant time delay," IEEE Transactions on Robotics, vol. 22, no. 2, pp. 269-281, apr 2006.

[22] E. Nuño, R. Ortega, N. Barabanov, and L. Basañez, "A globally stable PD controller for bilateral teleoperators," IEEE Transactions on Robotics, vol. 24, no. 3, pp. 753-758, jun 2008 .

[23] I. Polat and C. W. Scherer, "Stability analysis for bilateral teleoperation: An IQC formulation," IEEE Transactions on Robotics, vol. 28, no. 6, pp. 1294-1308, dec 2012.

[24] I. Polat, "Robustness analysis and controller synthesis for bilateral teleoperation systems via IQCs," Phd Thesis, TU Eindhoven, 2014.

[25] H. Tugal, J. Carrasco, and M. Maya-Gonzalez, "Teleoperation with memoryless, monotone, and bounded environments: A Zames-Falb multiplier approach," in European Control Conference, ECC, Linz, 2015, pp. $1968-1973$

[26] R. O'Shea, "An improved frequency time domain stability criterion for autonomous continuous systems," IEEE Transactions on Automatic Control, vol. 12, no. 6, pp. 725-731, dec 1967.

[27] G. Zames and P. L. Falb, "Stability conditions for systems with monotone and slope-restricted nonlinearities," SIAM Journal on Control, vol. 6, no. 1, pp. 89-108, 1968.

[28] J. Carrasco, M. C. Turner, and W. P. Heath, "Zames-Falb multipliers for absolute stability: From O'Shea's contribution to convex searches,' European Journal of Control, vol. 28, pp. 1-19, mar 2016.

[29] H. Morimitsu and S. Katsura, "Circle theorem-based realization of nonlinear force control for teleoperation under time delay," in IEEE International Conference on Mechatronics (ICM), Nagoya, 2015, pp. 562-567.

[30] M. Zheng, Z. Mao, K. Li, and M. Fei, "Quadratic separation framework for stability analysis of a class of systems with time delays," Neurocomputing, vol. 174, pp. 466-474, sep 2016.

[31] B. Anderson and S. Vongpanitlerd, Network analysis and synthesis: a modern systems theory approach. Prentice-Hall Englewood Cliffs, NJ, 1973.

[32] C.-Y. Kao, A. Megretski, U. T. Jonsson, and A. Rantzer, "A MATLAB toolbox for robustness analysis," in International Symposium on Computer Aided Control Systems Design. IEEE, 2004, pp. 297-302.

[33] T. Chen, "Global exponential stability of delayed Hopfield neural networks," Neural Networks, no. 14.8, pp. 977-980, 2001.

[34] A. Megretski and A. Rantzer, "System analysis via integral quadratic constraints," IEEE Transactions on Automatic Control, vol. 42, no. 6 , pp. 819-830, jun 1997.

[35] M. Jun and M. G. Safonov, "IQC robustness analysis for time-delay systems," International Journal of Robust and Nonlinear Control, vol. 11, no. 15 , pp. 1455-1468, dec 2001.

[36] A. Rantzer, "On the Kalman Yakubovich Popov lemma," Systems \& Control Letters, vol. 28, no. 1, pp. 7-10, 1996.

[37] E. Feron, "Analysis of robust $\mathrm{H} 2$ performance using multiplier theory," SIAM Journal on Control and Optimization, vol. 35, no. 1, pp. 160-177, 1997.

[38] J. Carrasco, M. Maya-Gonzalez, A. Lanzon, and W. P. Heath, "LMI searches for anticausal and noncausal rational Zames-Falb multipliers," Systems \& Control Letters, vol. 70, pp. 17-22, aug 2014. 
[39] H. Pfifer and P. Seiler, "An overview of integral quadratic constraints for delayed nonlinear and parameter-varying systems," arXiv:1504.02502, 2015.

[40] — " "Integral quadratic constraints for delayed nonlinear and parameter-varying systems," Automatica, vol. 56, no. January, pp. 36-43, 2015.

[41] C.-Y. Kao and A. Rantzer, "Stability analysis of systems with uncertain time-varying delays," Automatica, vol. 43, no. 6, pp. 959-970, jun 2007.

[42] X. Chen and J. T. Wen, "Robustness analysis for linear time-invariant systems with structured incrementally sector bounded feedback nonlinearities," Applied Mathematics and Computer Science, vol. 6, pp. 623648, 1996

[43] J. Veenman, C. W. Scherer, and H. Köroglu, "Robust stability and performance analysis based on integral quadratic constraints," European Journal of Control, in press, 2016.

[44] H. Kwakernaak and R. Sivan, Modern Signals and Systems. Prentice Hall, 1991.

[45] J. Veenman and C. Scherer, "IQC synthesis with general dynamic multipliers," International Journal of Robust and Nonlinear Control, vol. 24, no. 17, pp. 3027-3056, 2014.

[46] J.-P. Richard, "Time-delay systems: An overview of some recent advances and open problems," Automatica, vol. 39, no. 10, pp. 1667-1694, oct 2003.

[47] M. Jun and M. G. Safonov, "Rational multiplier IQCs for uncertain timedelays and LMI stability conditions," IEEE Transactions on Automatic Control, vol. 47, no. 11, pp. 1871-1875, 2002.

[48] M. Fu, H. Li, and S. Niculescu, "Robust stability and stabilization of time-delay systems via integral quadratic constraint approach," in Stability and control of time-delay systems. Springer Berlin Heidelberg, 1998, pp. 101-116.

[49] J. Lofberg, "YALMIP: A toolbox for modeling and optimization in MATLAB," in IEEE International Symposium on Computer Aided Control Systems Design, Taipei, sep 2004, pp. 284-289.

[50] Q. L. Han, "Absolute stability of time-delay systems with sectorbounded nonlinearity," Automatica, vol. 41, no. 12, pp. 2171-2176, 2005.

[51] M. Wu, Z. Y. Feng, and Y. He, "Improved delay-dependent absolute stability of Lur'e systems with time-delay," International Journal of Control, Automation and Systems, vol. 7, no. 6, pp. 1009-1014, 2009.

[52] A. Kazemy and M. Farrokhi, "Robust absolute stability analysis of multiple time-delay Lure systems with parametric uncertainties," Asian Journal of Control, vol. 15, no. 1, pp. 203-213, 2013.

[53] H. Zeng, S. Xiao, B. Qin, and H. Xiao, "Improved conditions for absolute stability of Lur'e systems with time-delay," in Third International Conference on Intelligent System Design and Engineering Applications. IEEE, jan 2013, pp. 862-865.

[54] S. Tarbouriech and M. Turner, "Anti-windup design: an overview of some recent advances and open problems," IET Control Theory \& Applications, vol. 3, no. 1, pp. 1-19, jan 2009.

[55] M. Turner, M. Kerr, and I. Postlethwaite, "On the existence of multipliers for MIMO systems with repeated slope-restricted nonlinearities," in ICCAS-SICE. IEEE, 2009, pp. 1052 - 1057.

[56] D. E. Rivera, M. Morari, and S. Skogestad, "Internal model control: PID controller design," Industrial \& engineering chemistry process design and development, vol. 25, no. 1, pp. 252-265, 1986. 